التحليل غير الخطي للصفائح الخرسانية المسلحة السميكة تحت تأثير الأحمال التكرارية والتسخين الاوري

$$
\text { أ.د. عبد ألحكيم حامد أحمد قسم الهنسة المدناصة / جامعة الموصل حكت علي الياس الجرجزي }
$$

تثمل هذه الدراسة تأثير التسخين الدوري والأحمال التكراريةفي السلوك ألإنشائنائي للصفائح الخراسانية

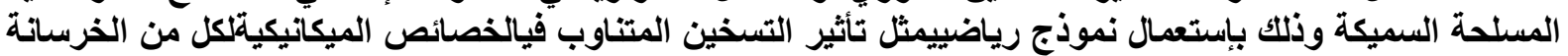

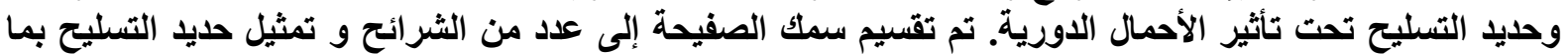

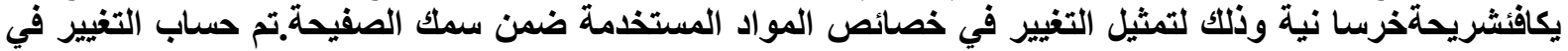

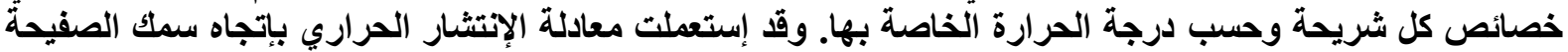

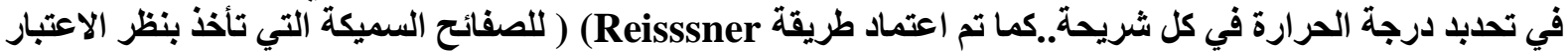

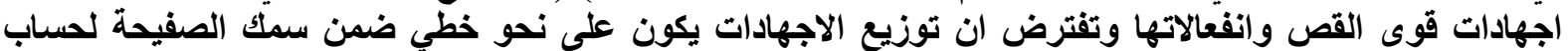

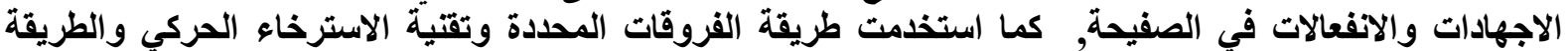

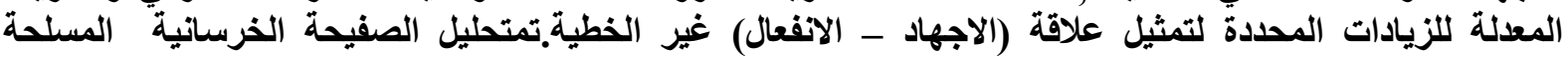

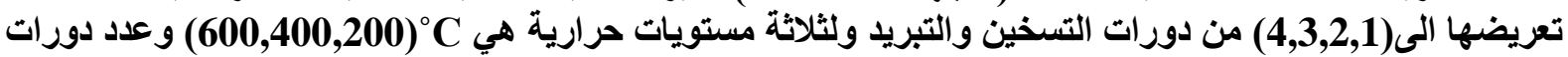

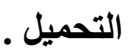

\title{
Nonlinear Analysis of Reinforced Concrete Thick Plates Under Effect of RepeatedLoading and Cyclic Heating
}

\author{
Dr. A.H. AHMAD (Professor) $\quad$ H. A. AL - Jarjery \\ Civil Engineering / University of Mosul
}

\begin{abstract}
This study includes the effect of cyclic heating and repeated loading on the behavior of reinforced concrete thick plates by using mathematical models to represent the effect of cyclic heating on mechanical properties of concrete and steel, and other models to represent the behavior of both concrete and steel under th effect of cyclic loads.

Plate thickness has been divided into several layers; steel was represented as an equivalent concrete layer. The material properties in each layer of the plate thickness are evaluated according to its temperature. One dimensional heat equation has been used to describe heat distribution through the layers

Reissener's theory that account for shear strain and transfer of shear forces and assumes that stress distribution across plate thickness is linear has been used to analyze the plates. The finite difference method and dynamic relaxation technique are used to calculate the stress and strain in the plate; also modified increment method is used to represent stress-strain nonlinear relationship.

Reinforced concrete plates have been analyzed in the ordinary temperature and after heating and cooling for three different temperatures $(200,400,600){ }^{\circ} \mathrm{C}$ with different Cyclic Loading of $(1,2,3,4)$ cycles .
\end{abstract}

Keywords: Nonlinear analysis, Reinforced concrete, Thick plates, Repeated load, High Temperature. 


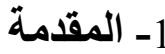

تُعد الصفائح الخرسة المُنية المسلحة من العناصر الإنشائية الهامة ونوجد في المنشآت الهندسية بشكل عام والمباني بشكل خاص. وقد تتعرض هذه المنشآت لأنواع مختلفة من الأحمال مثل الأحمال الدورية المتكررة وهي الأحمال التي تترواح بين الصفر

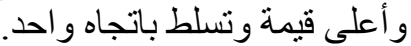

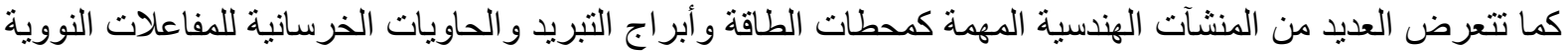

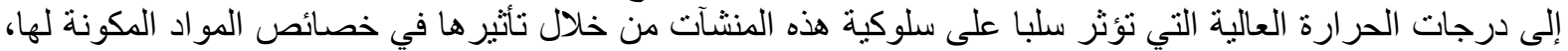

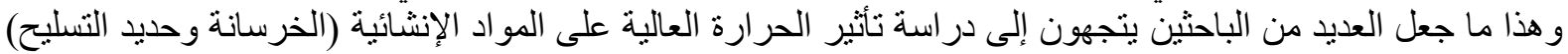
وبالتالي على سلوك العنصر الإنشائي.

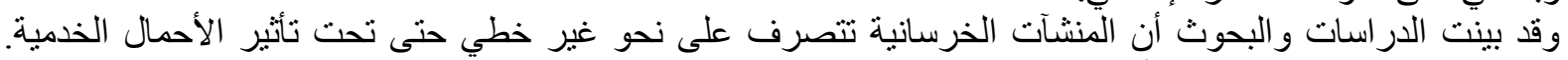
وينتج السلوك غير الخطي للمنشأ جراء السلوك غير الخطي المئ المادي ( Material Nonlinearly) المتمنل بعلاقة (الإجهاد ـ الانفعال) غير الخطية لكل من حديد التسليح و الخرسانة و هذا ما جعل التحليل غير الخطي أمرا لابد منه لتمثيل سلوك المنشآت الهندسية بشكل دقيق.

في هذه الدراسة تم استخدام طريقة الفرو قات المحددة وطريقة ألاسترخاء ألديناميكي لتحليل الصفائح الخرسانية السميكة

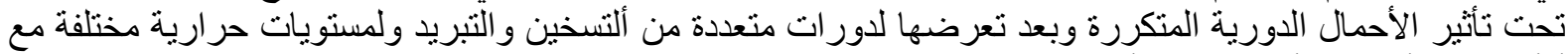

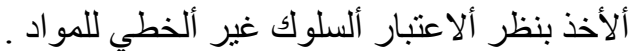

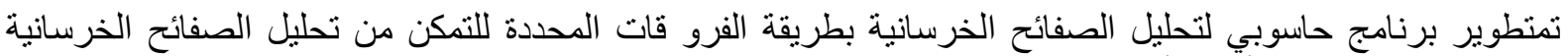

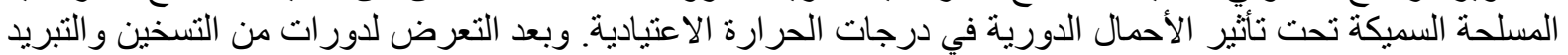

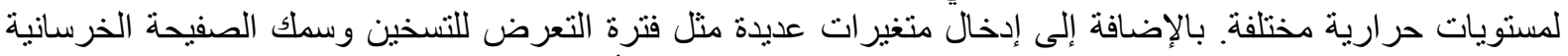

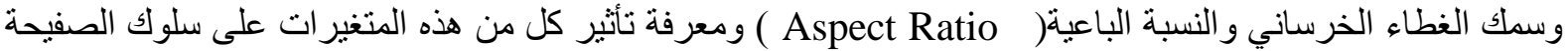
عند الدرجات الحر ارة العالية والاعتيادية.

في عام (2002) أجرى الباحثون (Tolentino وجماعته [1] دراسة عملية على نماذج خرسانية أسطوانية الثكل بأبعاد

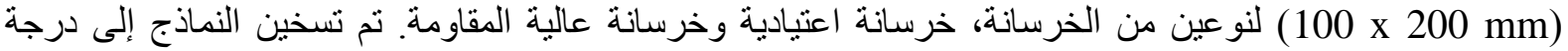

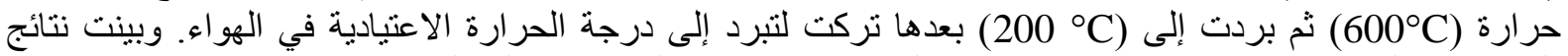

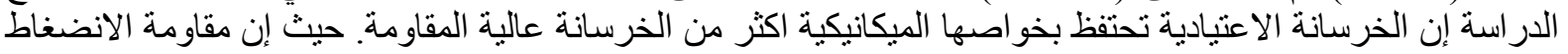

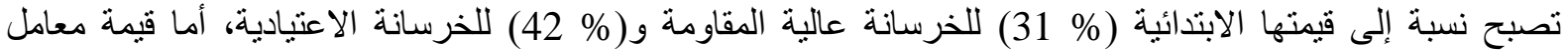

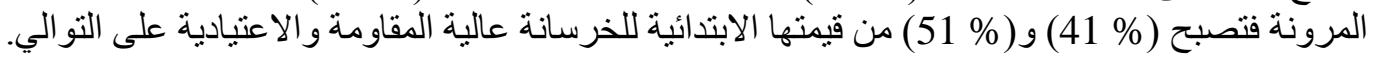

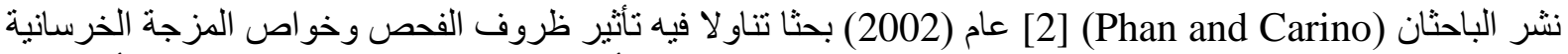

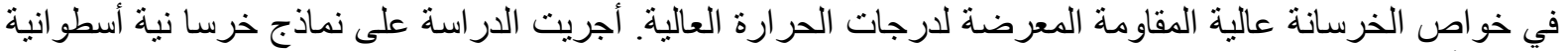

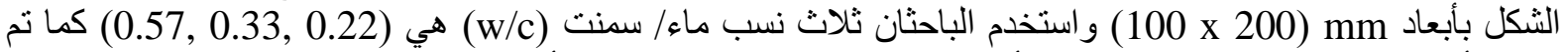

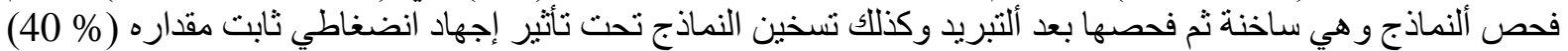

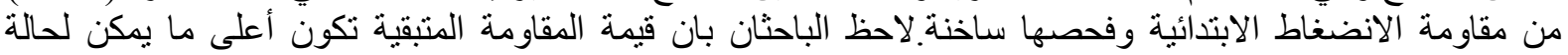

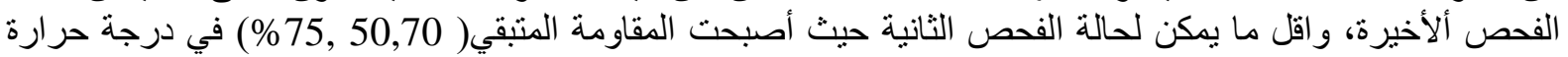

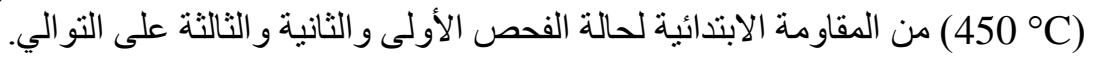

درس الباحثون (Cheng وجماعته) [3] عام (2004) سلوكية الخرسانة عالية المقاومة الاعتيادية (Plain Concrete)

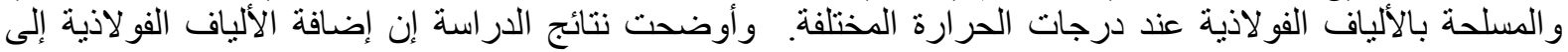

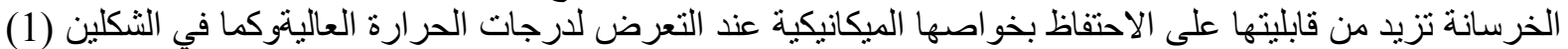

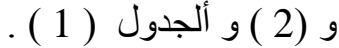
تناول الباحثون (AL - Tayyib وجماعته) [4] نتأثير التسخين المتكرر في الخرسانة الحاوية على الركام المحلي المنوفر

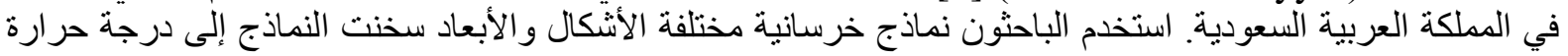

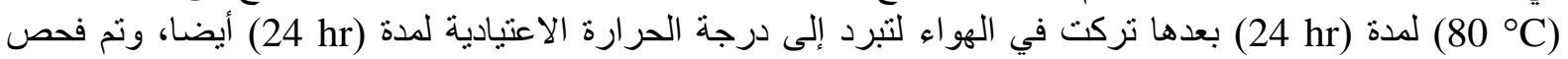
النماذج بعد تعريضها إلى (20) (20) (90) and 60, 


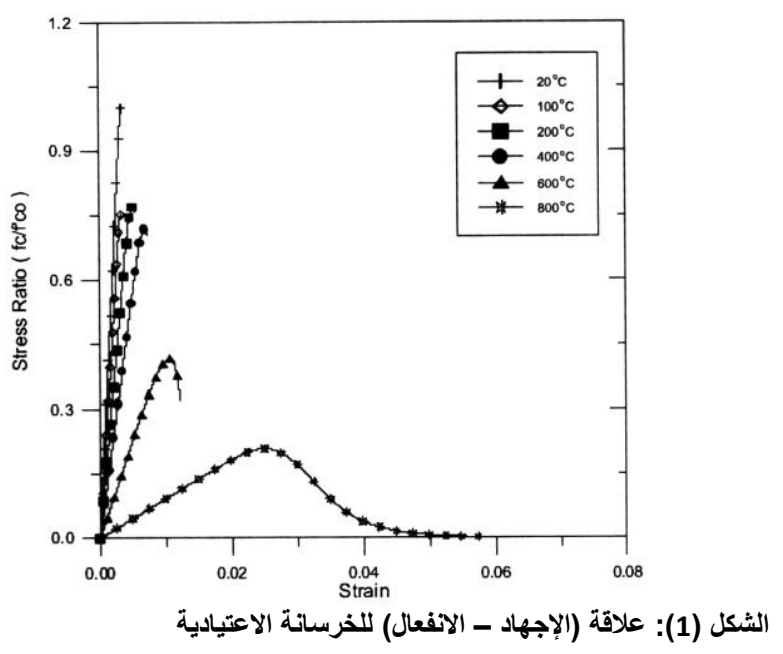

[3 [3 [3يات حرارية مختلفة

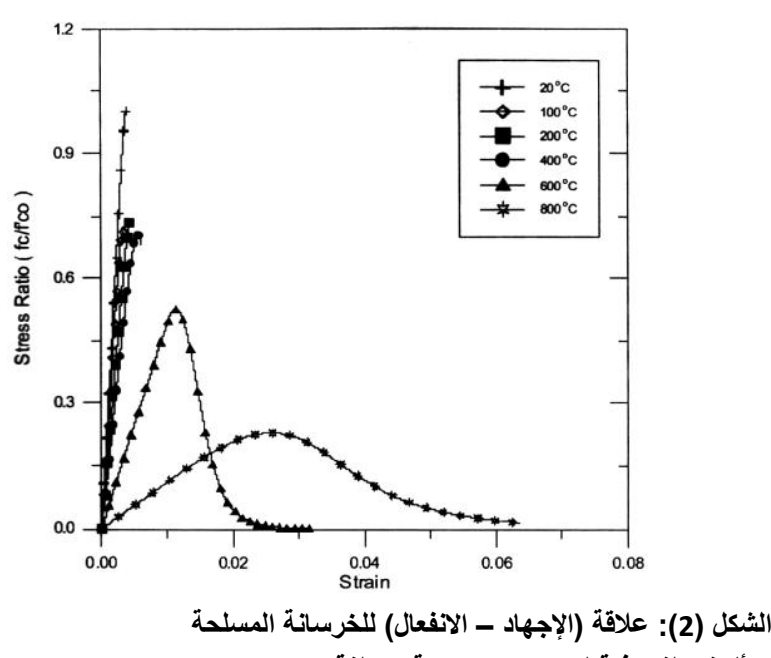

بالألياف الفو لاذية لمستويات حرارية مختلفة [3 الأفعال

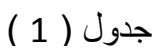

\begin{tabular}{|c|c|c|c|c|}
\hline \multicolumn{2}{|c|}{ خرسانة ليفية } & \multicolumn{2}{|c|}{ خرسانة اعنيادية } & \\
\hline $200 C^{\circ}$ & $400 \mathrm{C}^{\circ}$ & $200 \mathrm{C}$ & $400 \mathrm{C}$ & \\
\hline $81 \%$ & $48 \%$ & $42 \%$ & $76 \%$ & مقاو مةألإنضغاط \\
\hline $70 \%$ & $48 \%$ & $35 \%$ & $60 \%$ & معامل ألمرونة \\
\hline
\end{tabular}

وبينت الدراسة بان الخرسانة تفقد حوالي (\% 27 من من مقاومة انضغاطها بعد تعرضها إلى (90) دورة من التسخين

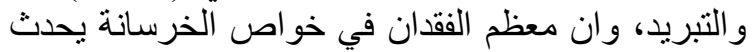

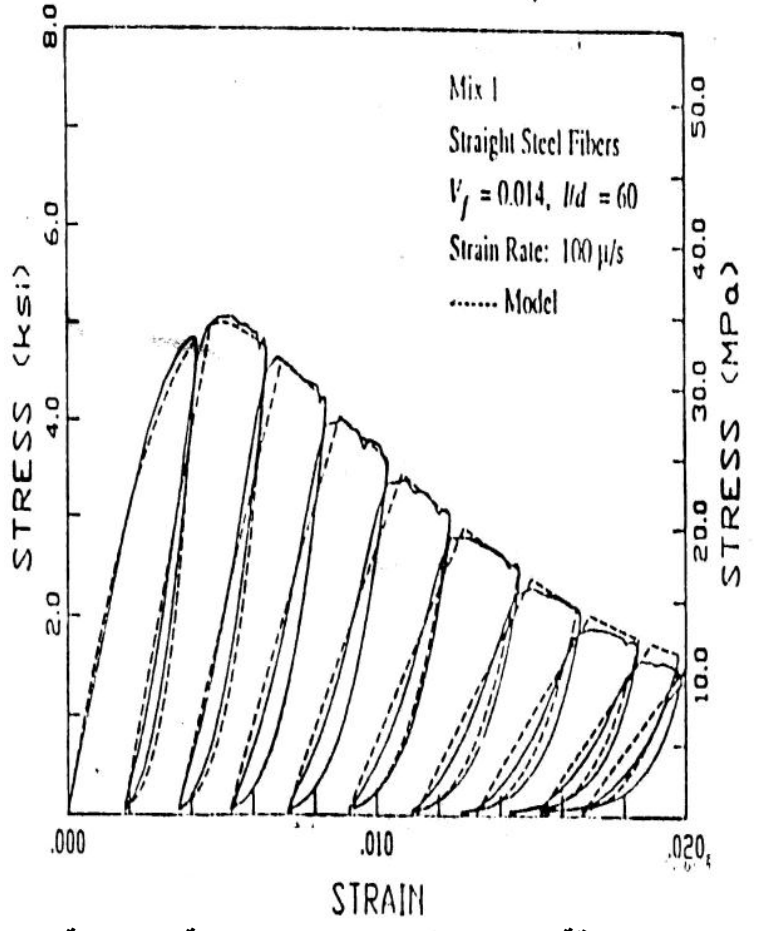

الثكل (3): علاقة (الإجهاد ـ الانفعال) للخرسانة المعرضة
في الدورات الثناثين الأولى من التسخين.

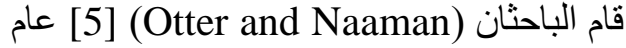

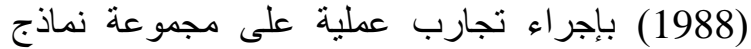
خرسانية أسطوانية ليفية بأبعاد

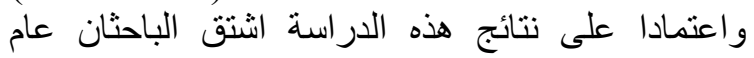

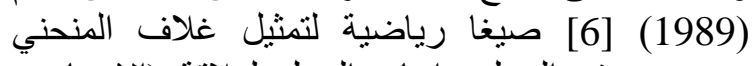

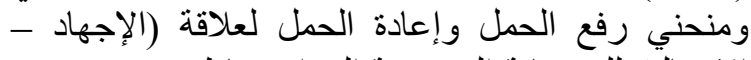

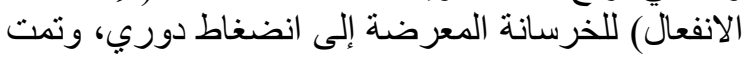

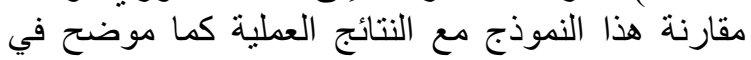

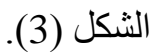

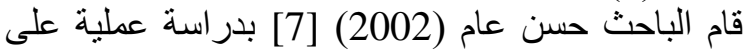

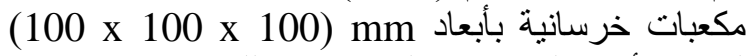

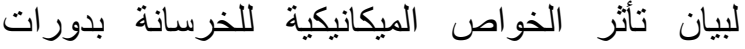
التسخين و التبريد بثلاثة مستويات حر ارية هي (600, 400, 200) ومدة التعرض اللتسخين مداها ساعة واحدة وبدون تسليط أب حمل في أثناء التسخين والتبريد واستتنج الباحث إن الخواص الميكانيكية للخرسانة تتأثر

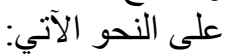
1.مقاومة الانضغاط: تتخفض مقاومة الانضغاط بعد الألئ الدورتين الأولى و الثانية عند درجة حرارة (200 (200 إلى (90, 84) \%ن قيمنها الأولية على التو الي وتتوقة (لته 
عن التأثر بعد الدورة الثانية. و عند درجة حرارة (600 C

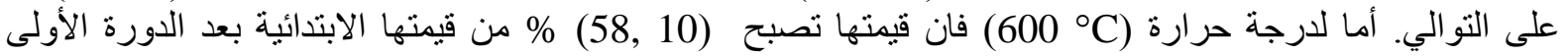

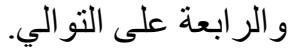

2.مقاومة الثد الانثطاري: لدرجة حرارة (65\% C قيمتها (65, 38) الدورتين الأولى و الر ابعة على التوالي.

3. معامل المرونة: تبدي قيمة معامل المرونة حساسية اكثر لدرجات الحر ارة ودورات التسخين و التبريد فعند درجة حرارة

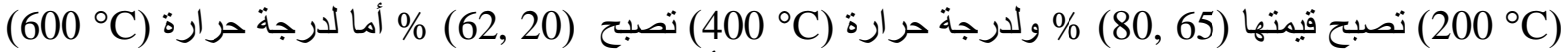
تتخفض قيمتها إلى $(40,10)$ \%من القيمة الابتدائية بعد الدورة الأولى و الرابعة على التو الي.

4. الانفعال الأقصى: تزداد قيمة الانفعال الأقصى بزيادة درجات الحرارة ودور ات التسخين و التبريد فبعد أربع دور ات من التص

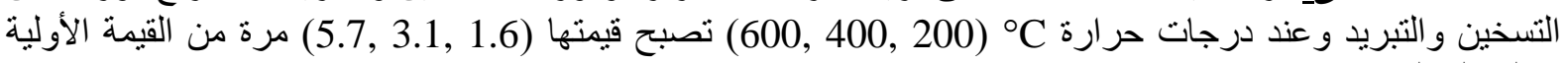
و وعلى التو الي.

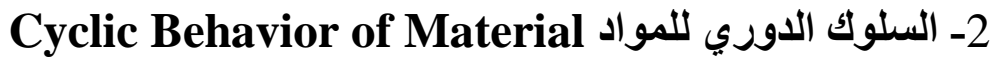

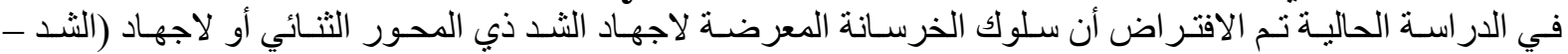
الانصغاط) لا يختلف عن سلوك الخر الخرسانة المعرضة لاجنة لاجهاد أحادية المحور.

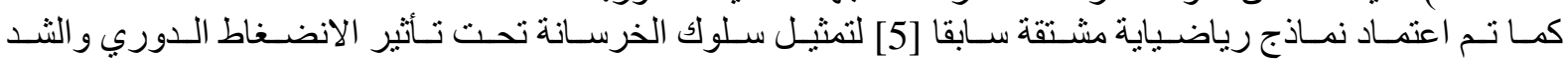
الدوري,وكذلك النموذج الدوري المعتمد لحديد التسليح.

1.2 علاقة (الإجهاد ـ الانفعال) للخرسانة تحت تأثير الانضغاط الدوري

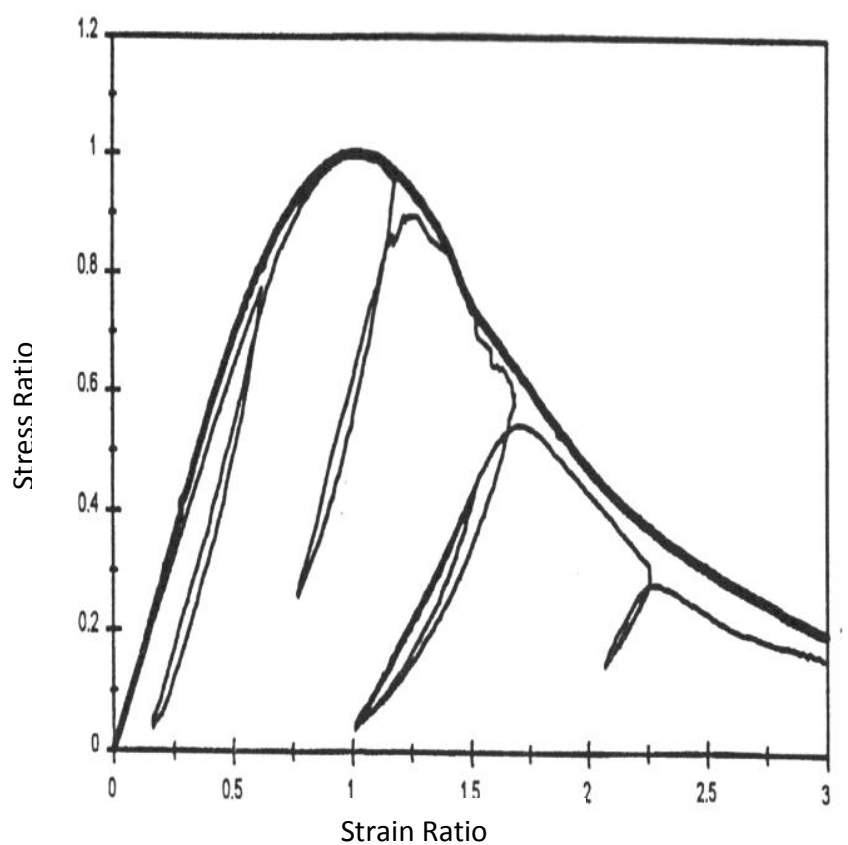

الثكل (4): غلاف المنحني تحت تأثير حمل تزايدي ودوري [8]

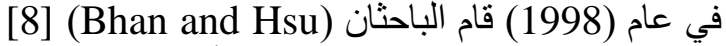
بدراسة عملية على نماذج خرسانية أسطوانية الثنكل

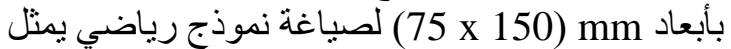

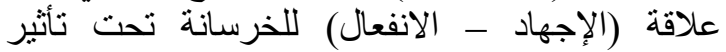

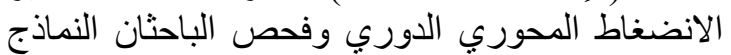

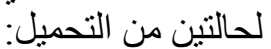

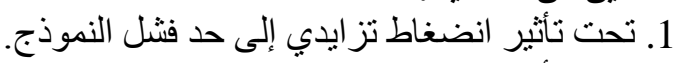
2. 1. تحت تأثير انضغاط دوري.

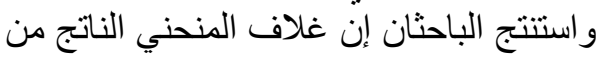

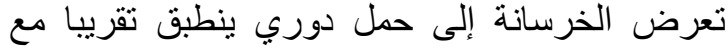
غلاف المنحني الناتج من الحمل التزئن ايدي كما موضح

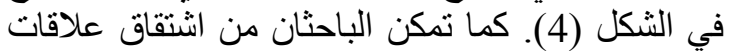

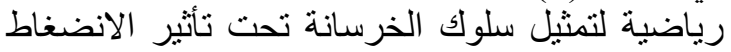
تم الاعتماد على النموذج الرياضي المقترح من قبل

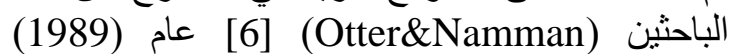
و الموضح في الثكل (5) لتمثيل سلوك الخرسانة تحت نأثير الانضغاط التكر اري. 


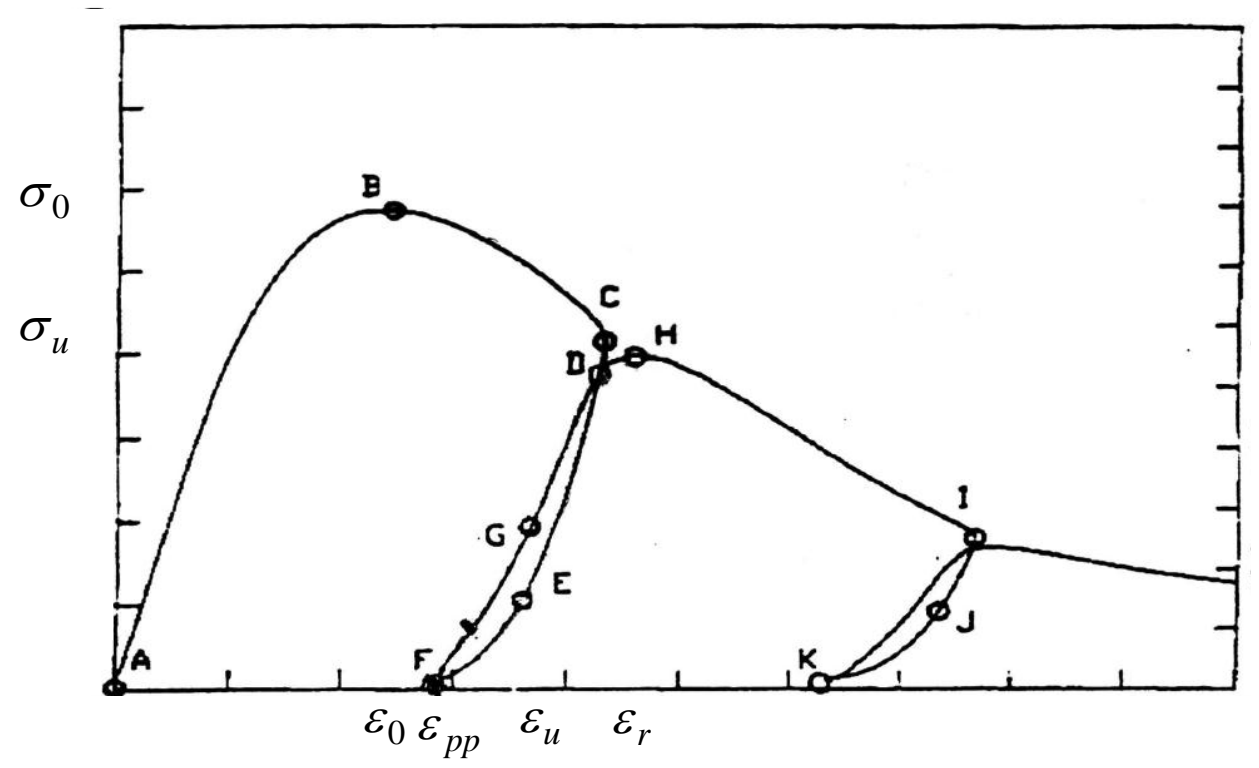

الشكل (5): السلوك الاوري للخرسانة في حالة رقع الحمل واعادته كليا [6]

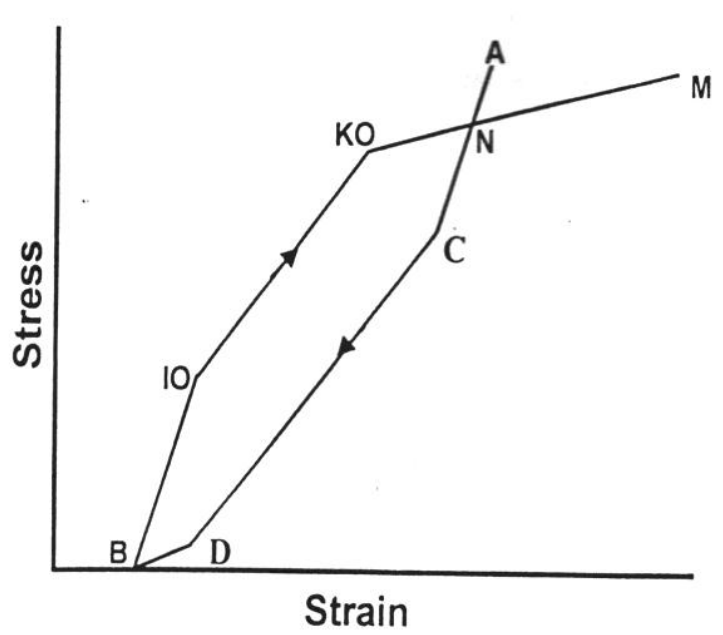

الثكل (6): سلوك الخرساني تحت تأثير شد دوري [9]

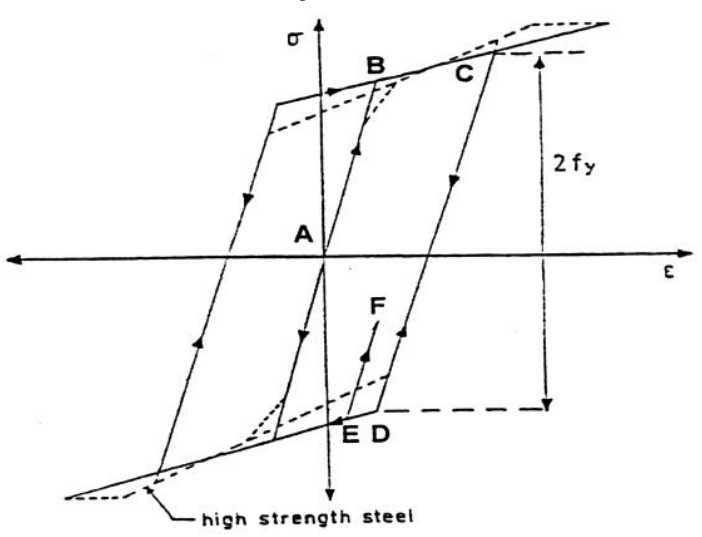

الثكل (7): سلوك حديد التسليح تحت تأثير الأحمال الدورية [10]
2.2: سلوك الخرسانة المعرضة لثد دوري Concrete Behavior Under Cyclic Tension تم الاعتماد على النموذج الذي اقترحه الباحثان [9] (Yankekevsky and Reinhardt ) الخرسانة عند تعرضهالأحمال شد تكراري.

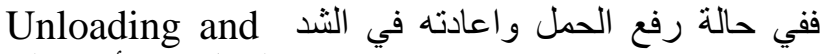
Reloading in Tension رفع الحمل تتوقف في النقطة (B) لتبدأ عملية إعادة الحمل و هكذا فان عمليتي رفع الحمل واعادته تتم في منطقة الثد الثد

\section{Steel Reinforcement}

2.3 حديد التسليح

اقترح الباحث (Chan) [10] عام (1982) النموذج المبين في الثكل (7) لتمثيل سلوك حديد التسليح تحت ناتثلثير الأحمال الدورية بلاحظ من الثنكل أن عملية رفع الحمل تبدأ من النقطة

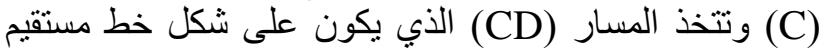

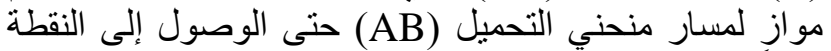

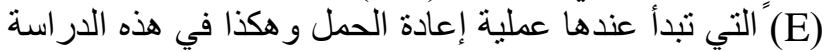

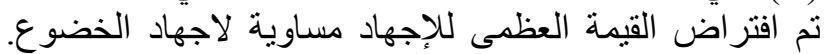

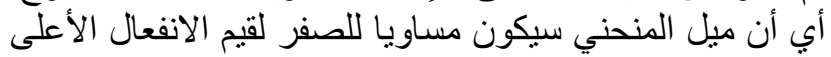
من الانفعال المقابلّ لاجهاد الخضوع وكما موضح لئح في الثنكل 
2.4

أوضحت نتائج الدراسة التي قام بها الباحث حسن [7] أن مقاومة الانضغاط تتناقص مع ارتفاع درجات الحرارة وزيادة

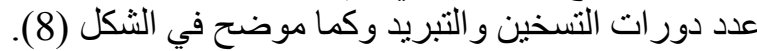

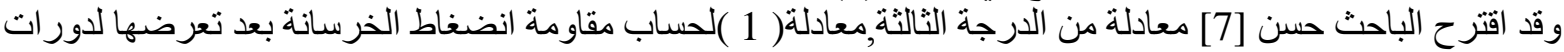
من التسخين والتبريد لمستويات حر ارية مختلفة و على النحو الآتي:

$f_{c t}=\frac{f_{c 20}}{100}\left(\begin{array}{l}X_{1}+X_{2} \times T+X_{3} \times T^{2} \times 10^{-3} \\ +X_{4} \times T^{3} \times 10^{-7}\end{array}\right)$

$X_{1}=96.67+6.972 C-3.392 C^{2}+0.452 C^{3}$

$X_{2}=\left(38.69-124.1 C+63 C^{2}-8.5 C^{3}\right) \times 10^{-3}$

$X_{3}=-3.533-3.776 C+2.78 C^{2}-0.4225 C^{3}$

$X_{4}=2.854-2.224 C+2.14 C^{2}-0.354 C^{3}$

مقاومة انضخاط الخرسانة بعد التسخين و التبريد: f $\left(\mathrm{mm}^{2} \mathrm{~N} /\right)$

( f $\mathbf{c 2 0}$

. (N/ $\left.\mathrm{mm}^{2}\right)$

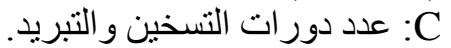

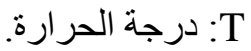

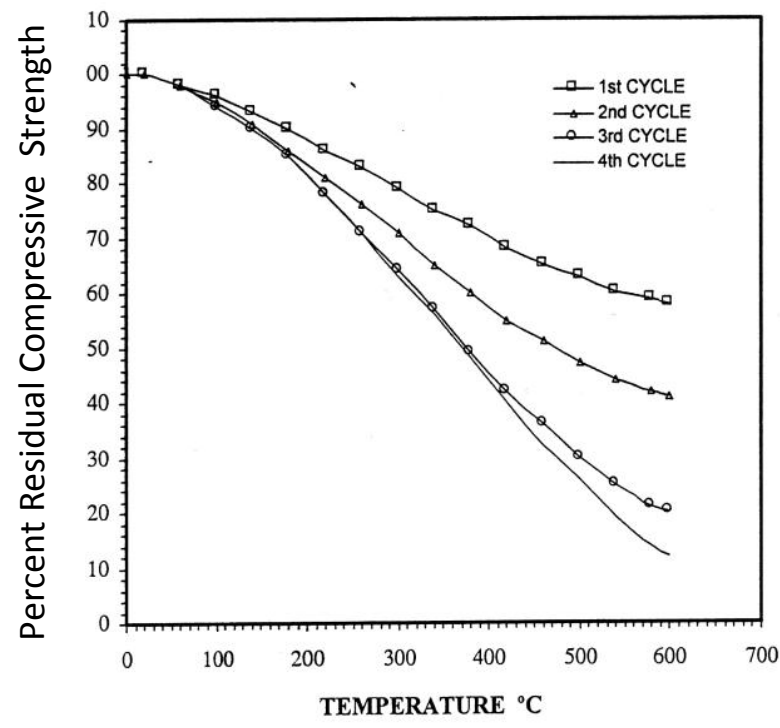

الثكل (8): تغير مقاومة الانضغاط للخرسانة مع ارتفاع درجات الحرارة

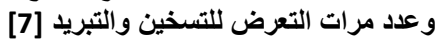

Modulus of Elasticity 2.5 في الدراسة الحالية تم الاعتماد على المعادلة ( 2 الألئ ) المقترحة من قبل مدونة (ACI) لحساب قيمة معامل المرونة الابتدائي و وعلى النحو الآتي [11] وكما موضح في الثكل (9).

$E_{c}=0.043 W^{3 / 2} \sqrt{f_{c}^{\prime}}$

حيث أن:

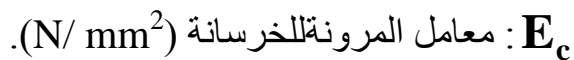
W : كثافة الخرسانة (

(N/ mm²) :

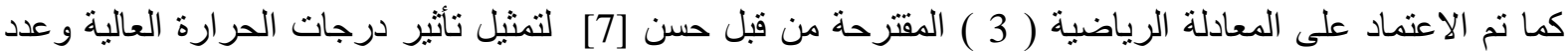
دورات التسخين والتبريد في قيمة معامل المرونة و الموضح بادئ بالثكل (10) و المعادلة المقترحة هي:

$\mathbf{E}_{\mathbf{T}}=\frac{\mathbf{E}_{20}}{\mathbf{1 0 0}}\left(\mathbf{X}_{1}+\mathbf{X}_{2}+\mathbf{X}_{3} \mathbf{T}^{2}+\mathbf{X}_{4} \mathbf{T}^{3}\right)$

$\mathrm{X}_{1}=87.05+20.712 \mathrm{C}-8.875 C^{2}+1.17 C^{3}$

$\mathrm{X}_{2}=0.375-0.7583 \mathrm{C}+0.3032 \mathrm{C}^{2}-0.0365 C^{3}$

$X_{3}=\left(-133.21+238.784 C-108.296 C^{2}+13.758 C^{3}\right) \times 10^{-5}$

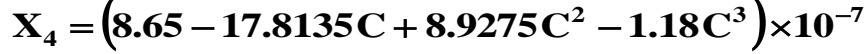

( E : E

(T) عند درجة حرارة (T)

معامل المرونة للخرسانة

(N/ mm²) $20 \mathrm{C}^{\mathrm{o}}$ ( عند درجة حرارة (N) 


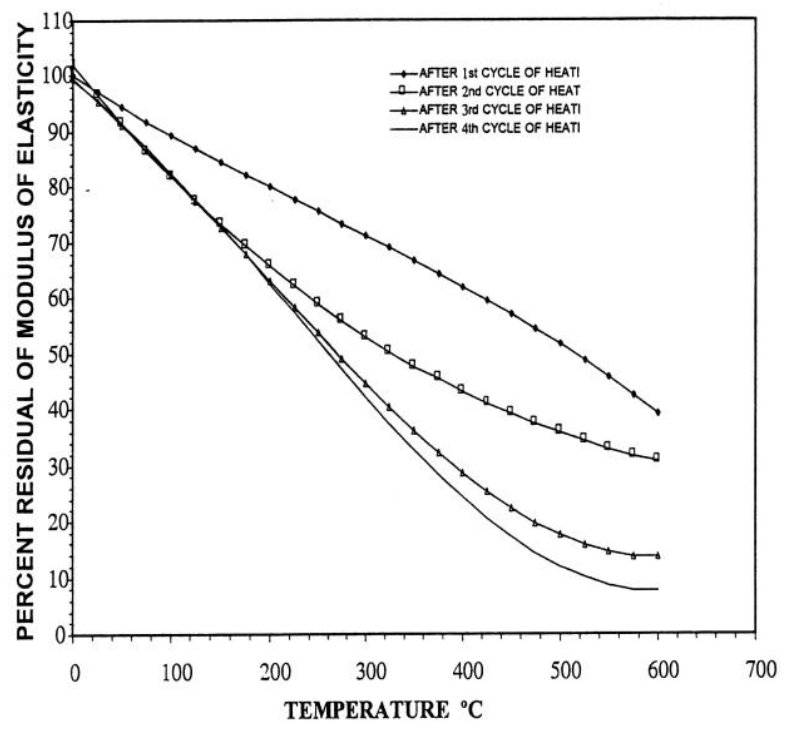

الثكل (10): تغير معامل المرونة للخرسانة مع ارتفاع درجات

[7] الحرارة وعدد مرات التعرض للتسخين

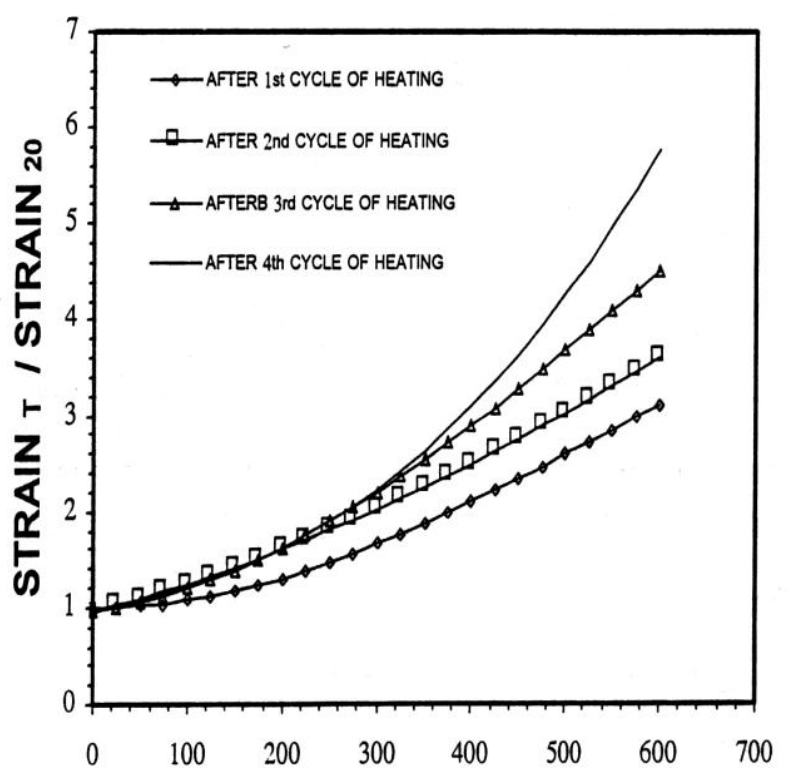

TEMPERATURE ${ }^{\circ} \mathrm{C}$

الشكل (11): تغير قيمة الانفعال القصوى مع تغير درجات

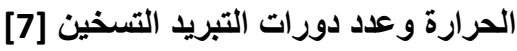

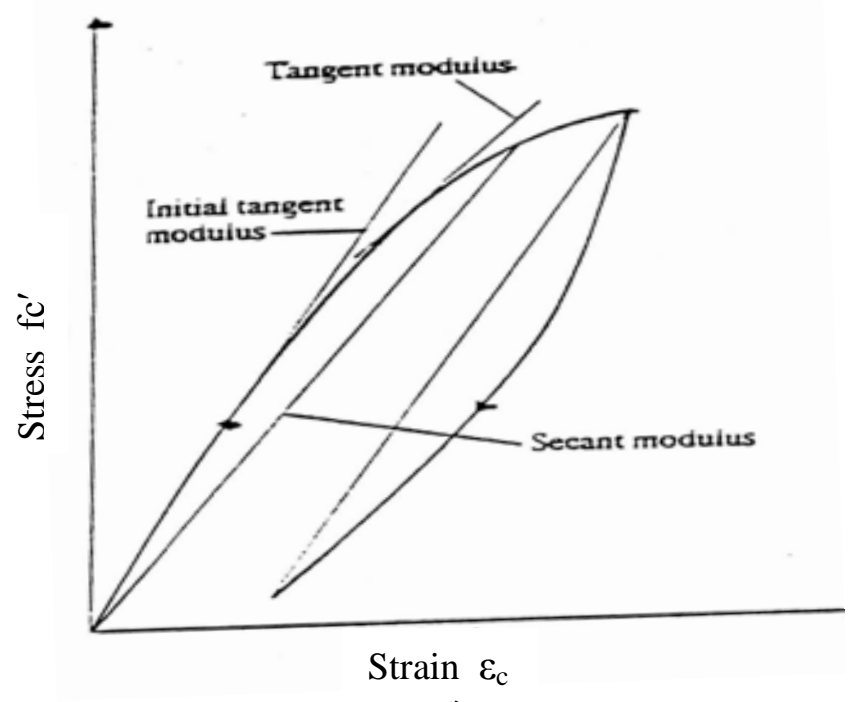

الثكل (9): معامل المرونة القاطع والمماسي [7]

\section{Strain}

لغرض حساب قيمة الانفعال بعد تعرض الخال الخرسانة إلى

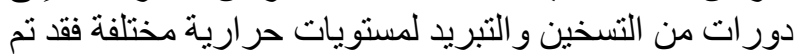

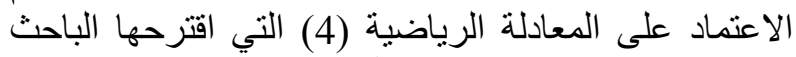
حسن شكل (11) و على النحو الآتي:

$\varepsilon_{p_{T}}=\varepsilon_{p_{20}}\left(X_{1}+X_{2} T+X_{3} T^{2}+X_{4} T^{3}\right)$

$X_{1}=1.252-0.3883 C+0.157 C^{2}-0.019535 C^{3}$ $X_{2}=\left(-137.776+220 C-85.556 C^{2}+10.707 C^{3}\right) \times 10^{-4}$ $X_{3}=\left(5.967-8.09 C+3.605 C^{2}-0.48 C^{3}\right) \times 10^{-5}$ $X_{4}=\left(52.96-77.57 C+36 C^{2}-5.1 C^{3}\right) \times 10^{-7}$

$$
\begin{aligned}
& \text { حيث أن: } \\
& \text { (T) } \\
& \text { (20) } \boldsymbol{\varepsilon}_{\mathbf{p}_{20}}
\end{aligned}
$$

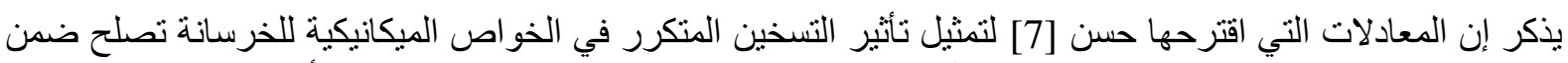

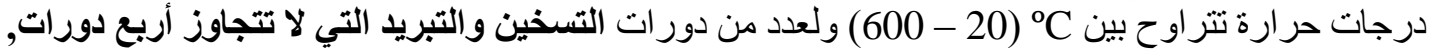




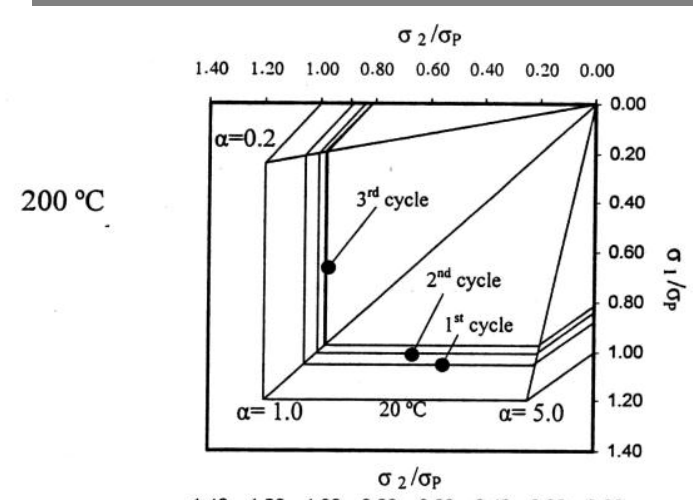

$\begin{array}{llllllll}1.40 & 1.20 & 1.00 & 0.80 & 0.60 & 0.40 & 0.20 & 0.00\end{array}$

$400^{\circ} \mathrm{C}$

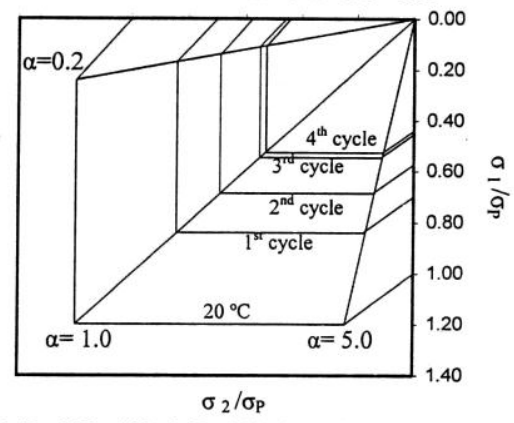

$600^{\circ} \mathrm{C}$

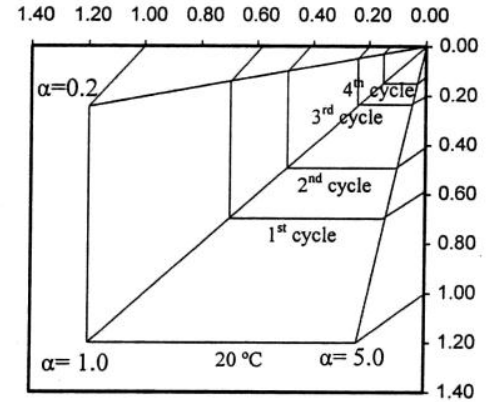

الثكل (12): مغلف الفثل ذو المحور الثنائي المقترح

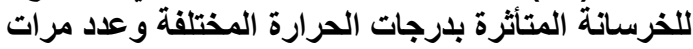

$$
\text { [7] التعرض للتسخين والتبرة }
$$

$$
\frac{\sigma_{p}}{\sigma_{o_{T}}}=1.2 \text { for } 0.2 \leq \alpha \leq 1.0
$$$$
\frac{\sigma_{\mathrm{p}}}{\sigma_{\mathrm{o}_{\mathrm{T}}}}=1+\frac{\alpha}{1.2-\alpha} \text { for } \alpha<0.2
$$$$
\frac{\sigma_{p}}{\sigma_{o_{T}}}=\frac{1.2}{\alpha} \text { for } 1.0 \leq \alpha \leq 5.0
$$$$
\frac{\sigma_{p}}{\sigma_{o_{T}}}=\frac{1}{\alpha}\left(1+\frac{1}{1.2 \alpha-1}\right) \text { for } \alpha>5.0
$$

3. ألعلاقات التكوينية:-

1.3.علاقة (الإجهاد_الانفعال)الثنائي المحور للخرسانة Biaxial Stress-Strain Relationship for concrete

اقترح الباحث (Liu) [11] علاقة رياضية (5) لتمثيل علاقة

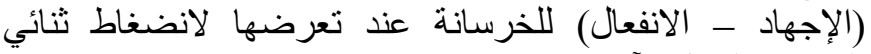

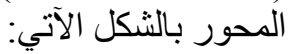

$$
\sigma=\frac{\varepsilon E}{(1-v \alpha)\left[1+\left[\frac{E}{E_{s}(1-v \alpha)}-2\right] \frac{\varepsilon}{\varepsilon_{p}}+\left[\frac{\varepsilon}{\varepsilon_{p}}\right]^{2}\right]}
$$
ع ع : قيم الإجهاد و الانفعال الأقصى على التوالي.

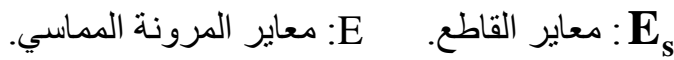
Q

2.3- المقاومة القصوى للخرسانة تحت تأثير الانضغاط ذي المحور الثنائي

Ultimate Strength of Concrete Under Biaxial Compression

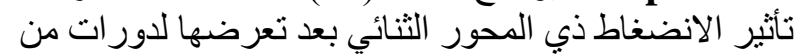

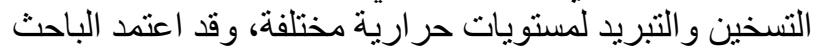

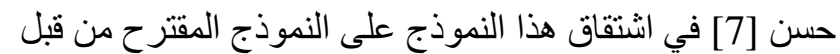

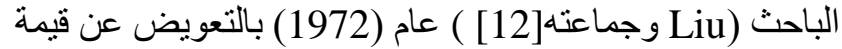

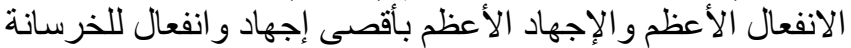

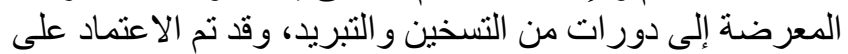
هذا النموذج في الدر اسة الحالية. و المعادلات التي تمثل الصيغة الرياضية لهذا النموذج هي:

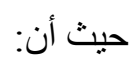

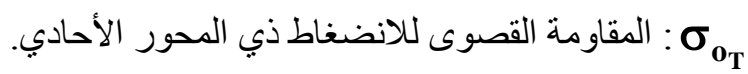
O 
3.3

\section{Strain at Ultimate Biaxial Compression Stress}

لتمثيل الانفعال عند الإجهاد الأعظم في درجات الحرارة العالية أو عند تعرض الخرسانة لدورات من التسخين و التبريد فقد $\varepsilon_{q}=-2500 \times 10^{-6} \times \zeta \quad$ (Major Direction)

$\varepsilon_{p}=\left(500+\zeta_{1} \sigma_{p}\right) \times 10^{-6} \quad$ (Minor Direction)

$\zeta_{1}=0.0266\left(500+\varepsilon q \times 10^{-6}\right)$

حيث أن:

$$
\text { ל : كعامل زيادة الانفعال الأقصى بتأثير الحرارة }\left(\frac{\boldsymbol{E}_{\mathbf{T}}}{\boldsymbol{\varepsilon}_{\mathbf{2 0}}}\right) .
$$

تم اقتر اح المعادلة (10) و المعادلة (11) استنادا إلى نتائج عملية نم إجراؤها ها على خرسانة بمقاومة انضغاط المقاط

ولغرض تعميم هذه المعادلات على الخرسانة بمستويات مختلفة لمقاومة الانضغاط أصبحت المانة المعادلة

بالصيغة الآتية:

$\varepsilon_{p}=\left[500+\zeta_{1} \times \sigma_{p} \times \frac{-31.33}{\sigma_{o}}\right] \times 10^{-6} \quad$ (Minor Direction)

حيث أن:

4- العلاقات المتحكمة بمصفوفات الخرسانة عند التحميل ذي المحور الثنائي Matrix Constitutive Relationship of Concrete in Biaxial Loading

حسب قانون هوك للمو اد المتجانسة الموحدة الخو اص يمكن كتابة المعادلات ألمتحكمة (13,14) بالصيغة الآتية [14] :

$\left[\begin{array}{c}\sigma_{1} \\ \sigma_{2} \\ \tau_{12}\end{array}\right]=\left[\begin{array}{ccc}\lambda^{\prime} E_{1 b}^{\prime} / E_{2 b}^{\prime} & \lambda^{\prime} v_{1} & 0 \\ \lambda^{\prime} v_{1} & \lambda^{\prime} & 0 \\ 0 & 0 & \frac{E_{1 b}^{\prime} E_{2 b}^{\prime}}{E_{1 b}^{\prime}+E_{2 b}^{\prime}+2 E_{2 b}^{\prime} v_{2}}\end{array}\right]\left\{\begin{array}{c}\varepsilon_{1} \\ \varepsilon_{2} \\ \gamma_{12}\end{array}\right\}$

$\left[\begin{array}{c}\tau_{13} \\ \tau_{23}\end{array}\right]=\left[\begin{array}{cc}E_{1 b}^{\prime} E_{c} / E_{1 b}^{\prime}+E_{c}+2 E_{c} v_{1} & 0 \\ 0 & E_{2 b}^{\prime} E_{c} / E_{2 b}^{\prime}+E_{c}+2 E_{c} v_{2}\end{array}\right]\left\{\begin{array}{l}\gamma_{13} \\ \gamma_{23}\end{array}\right\}$

$E_{1 b}^{\prime}=\frac{E\left(1-\left(\frac{\varepsilon_{1}}{\varepsilon_{p}}\right)^{2}\right)}{\left[1+\left[\frac{E}{E_{s}(1-v \alpha)}-2\right]\left[\frac{\varepsilon_{1}}{\varepsilon_{p}}\right]+\left[\frac{\varepsilon_{1}}{\varepsilon_{p}}\right]^{2}\right]^{2}}$

حيث أن القيم التالية في حالة الانضغاط ذي المحور الثنائي تكون:- 


$$
E_{2 b}^{\prime}=\frac{E\left(1-\left(\frac{\varepsilon_{2}}{\varepsilon_{p}}\right)^{2}\right)}{\left[1+\left[\frac{E}{E_{s}(1-v \alpha)}-2\right]\left[\frac{\varepsilon_{2}}{\varepsilon_{p}}\right]+\left[\frac{\varepsilon_{2}}{\varepsilon_{p}}\right]^{2}\right]^{2}}
$$

$$
\text { وفي حالة الثد ذي المحور الثنائي وحالة الثند - الانضغاط تكون:- }
$$

$E_{1 b}^{\prime}=E_{2 b}^{\prime}=E \quad$ Unixial Modulus of Elasticity

$\lambda^{\prime}=E_{1 b}^{\prime} /\left(E_{1 b}^{\prime} / E_{2 b}^{\prime}-v^{2}\right)$

\section{ConcreteStress - Strain Relations for Cracked}

5- علاقة (الإجهاد_الانفعال)للخرسانة المتثققة:-

تكون علاقة (الإجهاد - الانفعال) للخرسانة المعرضة إلى إجهاد الثد علاقة خطية إلى حين وصول الإجهاد إلى قيمة

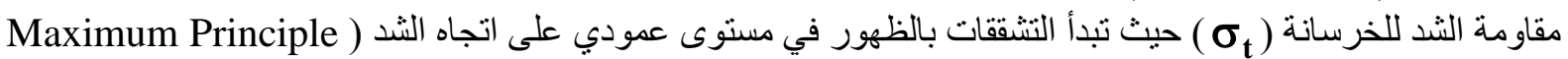
(Tensile Stress

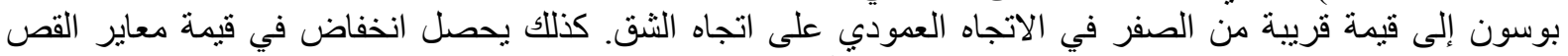
و وندها يمكن كتابة علاقة (الإجهاد - الآفعال) بالثكل الآتي: الآني:

$$
\left[\begin{array}{c}
\sigma_{1} \\
\sigma_{2} \\
\tau_{12} \\
\tau_{13} \\
\tau_{23}
\end{array}\right]=\left[\begin{array}{ccccc}
0 & 0 & 0 & 0 & 0 \\
0 & C_{1} E & 0 & 0 & 0 \\
0 & 0 & G_{12}^{\prime} & 0 & 0 \\
0 & 0 & 0 & G_{13}^{\prime} & 0 \\
0 & 0 & 0 & 0 & G_{23}^{\prime}
\end{array}\right]\left\{\begin{array}{c}
\varepsilon_{1} \\
\varepsilon_{2} \\
\gamma_{12} \\
\gamma_{13} \\
\gamma_{23}
\end{array}\right\}
$$

حيث أن:

$$
\text { Cي }
$$$$
\text { : C } 1 \text { = }
$$

: تمثل قيمة معاير القص المخفضة.

\section{Slab Thermal Analysis}

$$
\text { 6- التحليل الحراري للصفائح }
$$

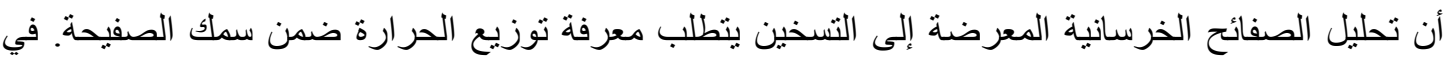

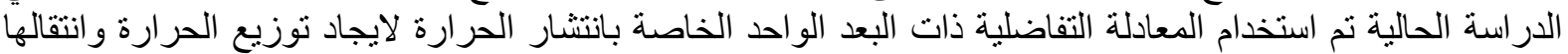

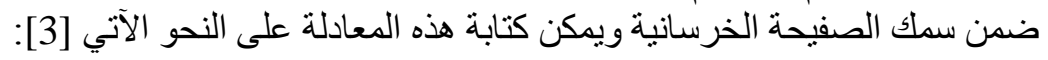

$K \frac{\partial^{2} T}{\partial X^{2}}=C_{p} \rho \frac{\partial T}{\partial \theta}$

حيث أن:

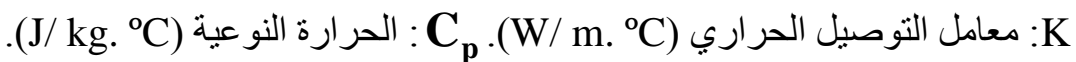

O 
ويمكن التعبير عن انتقال الحرارة من المستوي (n-1) إلى (n+1) بطريقة الفرو قات المحددة وعلى النحو الآتي:

$T_{n}^{t+1}=\left[1-2 \frac{\Delta \theta K}{C_{p} \rho \Delta X^{2}}\right] T_{n}^{t}+\frac{\Delta \theta K}{C_{p} \rho \Delta X^{2}}\left(T_{n-1}^{t}+T_{n+1}^{t}\right)$

Method of Analysisis

تكون محصلة العزومللصفائح الخرسانية ذات الخو اصل الدتجهة بالصيغة الآتية:

$$
\begin{aligned}
& {\left[\begin{array}{l}
M_{x} \\
M_{y} \\
M_{x y}
\end{array}\right]=\left[\begin{array}{lll}
D_{11} & D_{12} & D_{13} \\
D_{21} & D_{22} & D_{23} \\
D_{31} & D_{32} & D_{33}
\end{array}\right]\left\{\begin{array}{l}
K_{x} \\
K_{y} \\
K_{x y}
\end{array}\right\}} \\
& D_{11}=D_{x}, \quad D_{12}=v D_{11} \\
& D_{22}=D_{y}, \quad D_{33}=\frac{1-v}{2} D_{x y} \\
& D_{13}=D_{23}=0
\end{aligned}
$$

(Curvature) (Kثل قيم التحدب (C) $\left(K_{y}, K_{x y}\right)$

$$
\begin{aligned}
& K_{x}=\frac{\partial Q_{x}}{\partial x}=\frac{\partial^{2} w}{\partial x^{2}}-\frac{K}{G h} \frac{\partial Q_{x}}{\partial x} \\
& K_{y}=\frac{\partial Q_{y}}{\partial y}=\frac{\partial^{2} w}{\partial y^{2}}-\frac{K}{G h} \frac{\partial Q_{y}}{\partial y} \\
& K_{x}=\frac{\partial Q_{x}}{\partial y}=\frac{\partial Q_{y}}{\partial x}-2 \frac{\partial^{2} w}{\partial x \partial y}-\frac{K}{G h}\left[\frac{\partial Q_{x}}{\partial y}+\frac{\partial Q_{y}}{\partial x}\right]
\end{aligned}
$$

أما العلاقة بين قوى القص و انفعالات القص في حالة الصفيحة اتجاهية الخو اص فتكون بالثكل الآتي:

$$
\left[\begin{array}{l}
Q_{x} \\
Q_{y}
\end{array}\right]=\left[\begin{array}{ll}
G h_{11} & G h_{12} \\
G h_{21} & G h_{22}
\end{array}\right]\left\{\begin{array}{l}
\gamma_{x z} \\
\gamma_{y z}
\end{array}\right\}
$$

Finite Difference Method 1-7 طريقة الفروقات المحددة

ويتم الحل بتطبيق معادلات التوازن للصفيحة في كافة النقاط. في الدراسة الحالية نم حل معادلة التوازن الآتية بإستعمال طريقة الفرو قات المحددة:

$$
\frac{\partial^{2} M_{x}}{\partial x^{2}}-2 \frac{\partial^{2} M_{x y}}{\partial x \partial y}+\frac{\partial^{2} M_{y}}{\partial y^{2}}=-q
$$

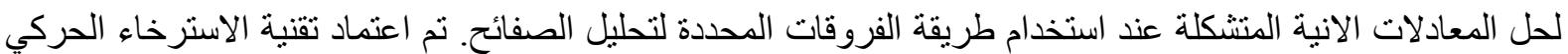

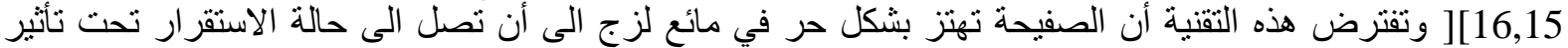

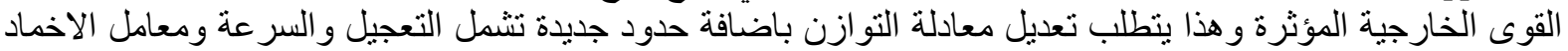
لتصبح المعادلة (26) بالثكل الآتي:

$$
-\frac{\partial^{2} M_{x}}{\partial x^{2}}-2 \frac{\partial^{2} M_{x y}}{\partial x \partial y}-\frac{\partial^{2} M_{y}}{\partial y^{2}}+D_{f} \frac{\partial w}{\partial t}+m \frac{\partial^{2} w}{\partial t^{2} 11}=q
$$




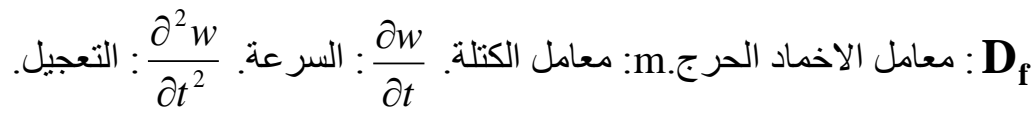

ولغرض تحويل المعادلة السابقة الى معادلة لا بعدية تم تمثيل كل عنصر من عناصر ها بالصيغة اللابعدية و على النحو

$$
\begin{array}{llll}
X=L \bar{X} & q=q_{o} \bar{q} & t=t_{o} \bar{t} & M_{x}=q_{o} l^{2} \bar{M}_{x} \\
M_{y}=q_{o} l^{2} \bar{M}_{y} & M_{x y}=q_{o} l^{2} \bar{M}_{x y} & w=\frac{q_{o} l^{4}}{D_{o}} \bar{w} & D_{f}=\frac{t_{o} D_{o}}{l^{4}} \bar{D}_{f} \\
m=\frac{t_{o}^{2} D_{o}}{l^{4}} \bar{m} & D=\bar{D} D_{o} &
\end{array}
$$

L Lول Lئ الصفيحة، و الرمز (-) لغرض التعبير عن القيمة اللابعدية. و الان يمكن كتابة المعادلة (26) بالصيغة اللابعدية على النحو الآتي:

$$
\frac{\partial^{2} \bar{M}_{x}}{\partial \bar{x}^{2}}-2 \frac{\partial^{2} \bar{M}_{x y}}{\partial \bar{x} \partial \bar{y}}-\frac{\partial^{2} \bar{M}_{y}}{\partial \bar{y}^{2}}+\bar{D}_{f} \frac{\partial \bar{w}}{\partial \bar{t}}+\bar{m} \frac{\partial^{2} \bar{w}}{\partial \bar{t}^{2}}=\bar{q}
$$

ويمكن التعبير عن السر عة عند كل زيادة زمن بالصبغة الآتية:

$$
\bar{w}_{(i) j}^{o}=\frac{1}{1+0.5 \bar{D}_{v}}\left[(1-0.5 \bar{D} v) \bar{w}_{(i) j}^{o}+\frac{\Delta \bar{t}}{\bar{m}}\left[\left[\frac{\partial^{2} \bar{M}_{x}}{\partial \bar{x}^{2}}\right]_{i}+2\left[\frac{\partial^{2} \bar{M}_{x y}}{\partial \bar{x} \partial \bar{y}}\right]_{i}+\left[\frac{\partial^{2} \bar{M}_{y}}{\partial \bar{y}^{2}}\right]+\bar{q}_{i}\right]\right]
$$

كما يمكن التعبير عن قيم الاود في الحالة المستقرة بالصيغة الآتبة:

$$
\sum_{K=1}^{K=j+0.5} \bar{w}_{(i) K}=\sum_{K=1}^{K=j-0.5} \bar{w}_{(i) K}+\bar{w}_{(i) j}^{o} \Delta \bar{t}
$$

$$
\begin{aligned}
& \text { حيث أن: } \\
& \text { : Dع } \\
& \text { J : الزيادة في الوقت. W0 }
\end{aligned}
$$

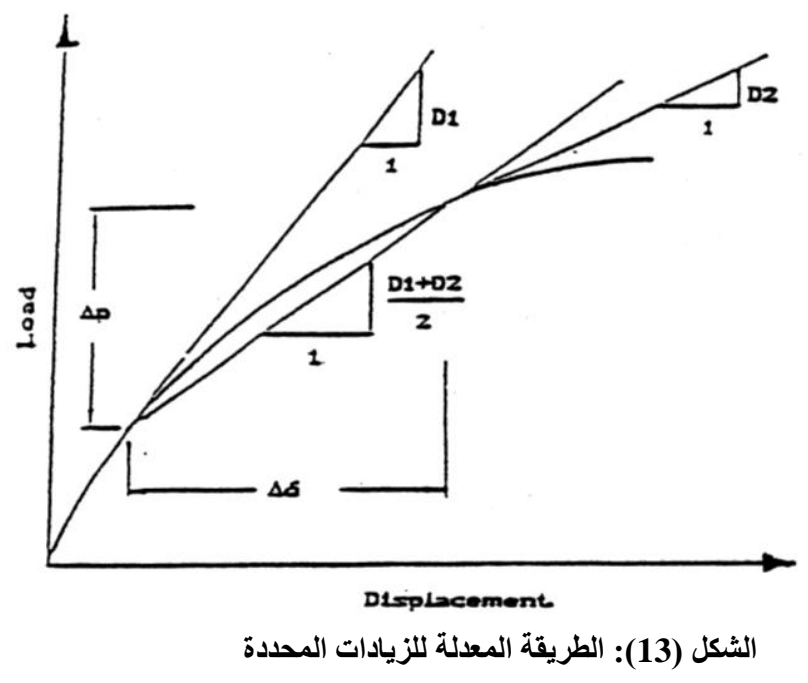

Modified 7.2 Increment Method

في الدراسة الحالية تم الاعتماد على الطريقة المعدلة

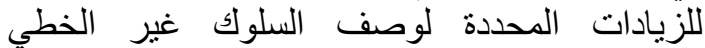
للصفائح الخرسانية. ويتم في هذه الطريقة الطيات حساب

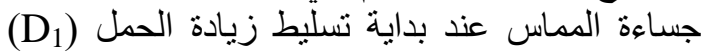

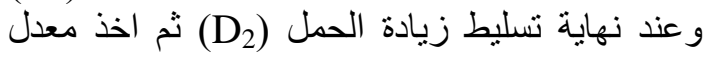
الجساءتين كما موضح في الثكل نيانة (13) (13) 


\section{Results and Discussion}

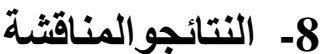

تأثير التسخين الدوري والأحمال الاورية يوضح الثكل (14) علاقة الحمل-الأود للصفيحة الخرسانية (S1) تحت تأثير الحمل التزايدي (Monotonic Load) في الحر ارة الاعتبادية وبعد التعرض لدورة و احدة من التسخين و التبريد لمستويات حرارية مختلفة، ويلاحظ من الثكل إن أداء

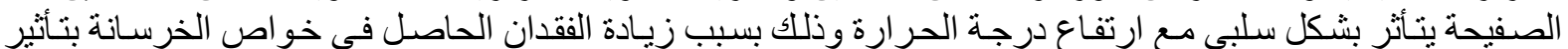

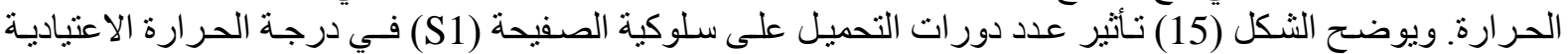
ويلاحظ بان جساءة الصفيحة تقل ويحصل انخفاض في تحملها ويعزى السبب إلى نمو التشققات في كتلة الخرسانة.

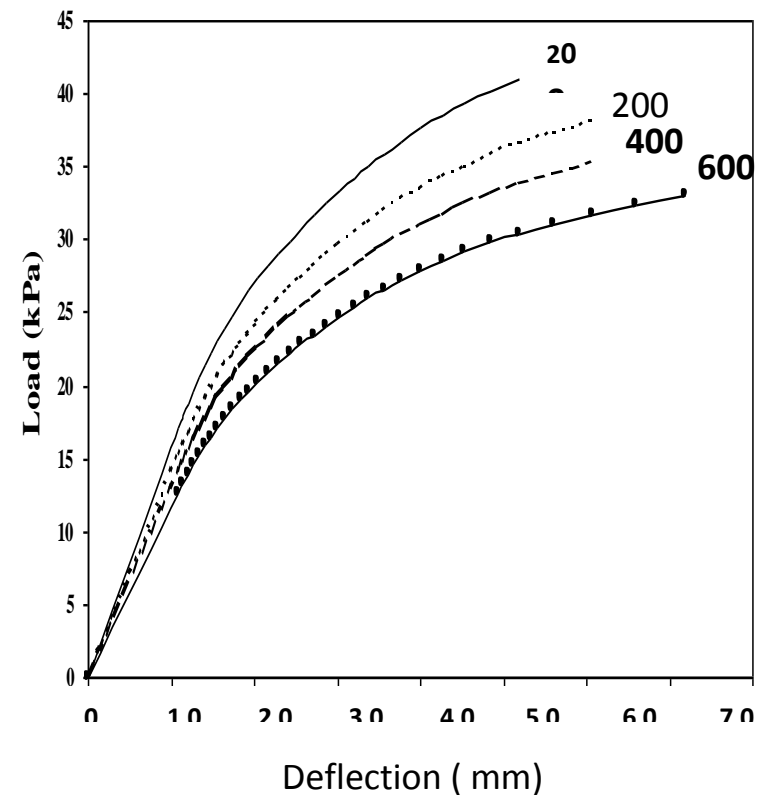

الثكل(14) علاقة الأودـالحمل للصفيحة الخرسانية S1 في درجة والقدئ الحرارة الاعتيادية وبعد دورة واحدة من الحيدة التسخين والتبريد لمستويات حرارية مختلفة

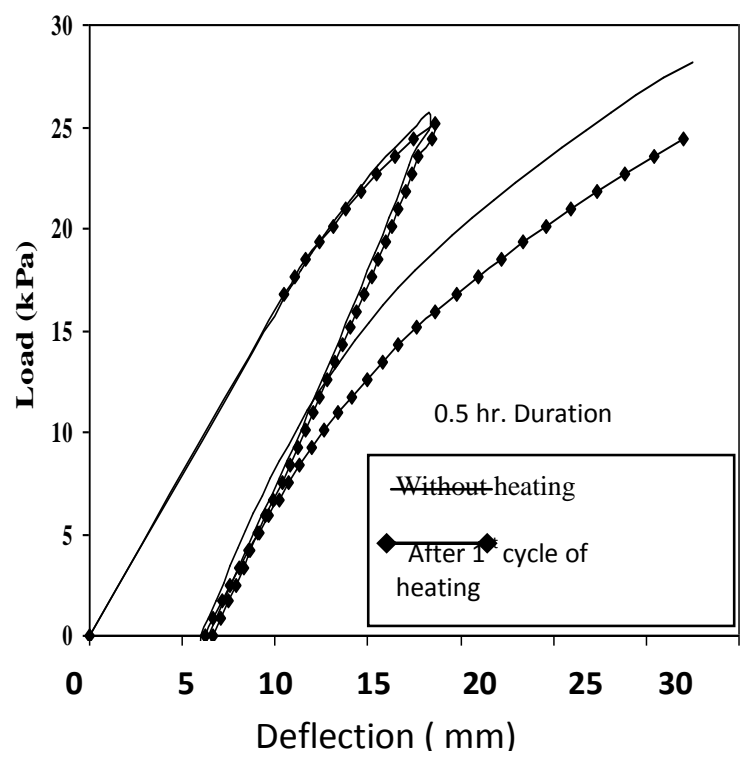

الثكل (16.a): علاقة (الاود - الحمل) للصفيحة S1

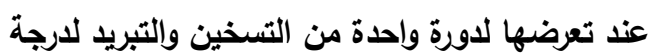

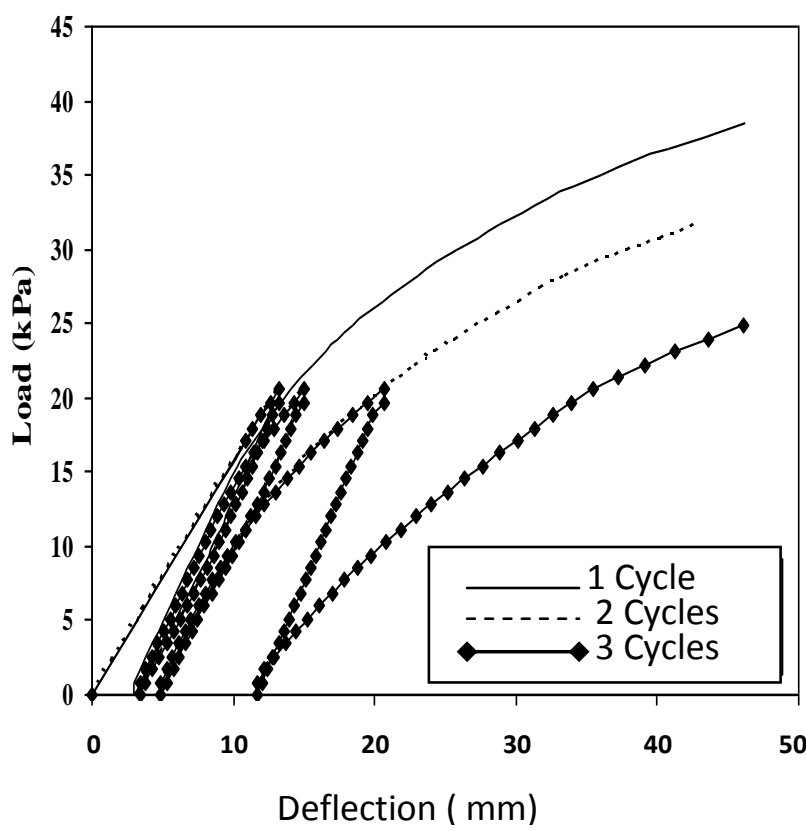

الثكل(15) تأثير دورات التحميل على سلوك الصفيحة الخرسانية S1 في

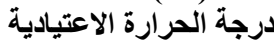

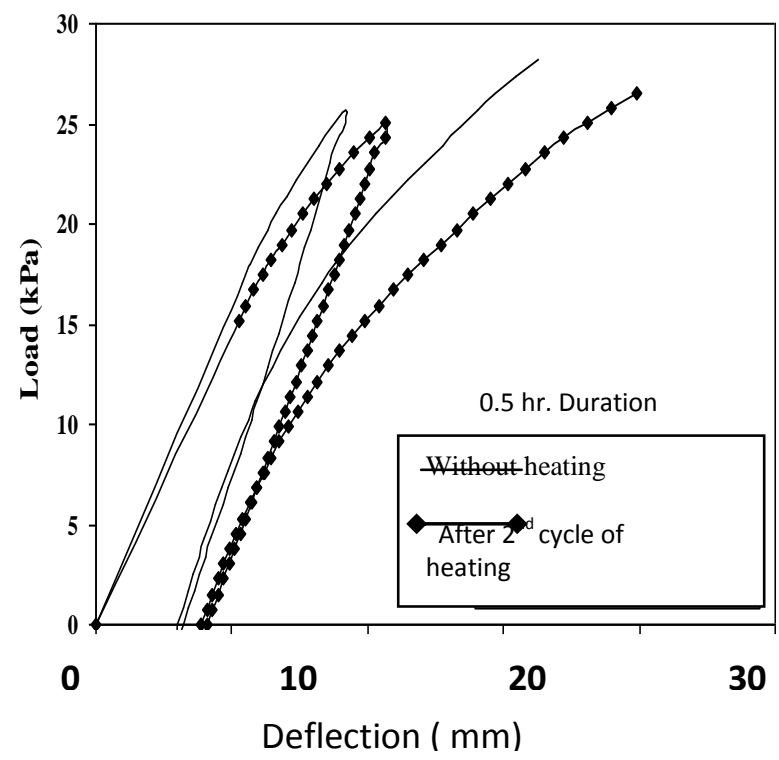

الثنكل (16.b): علاقة (الاود - الحمل) للصفيحة S1 عند

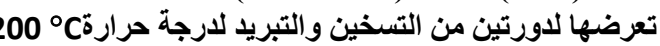




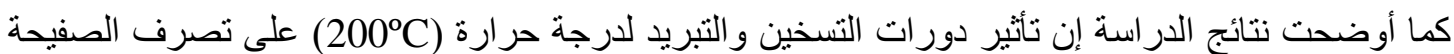

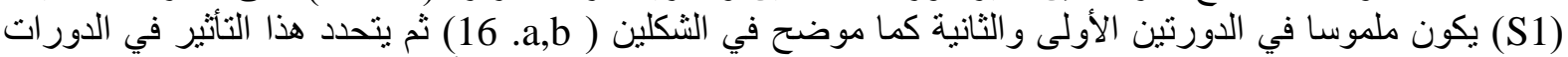

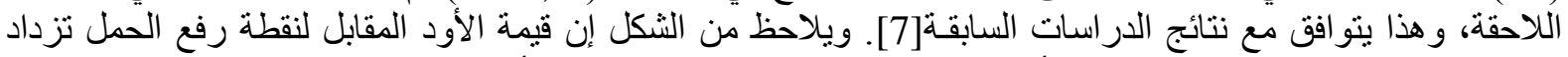

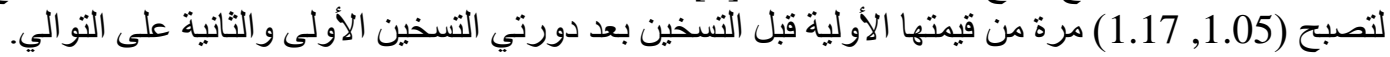

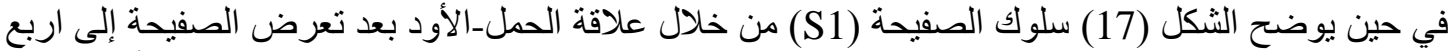

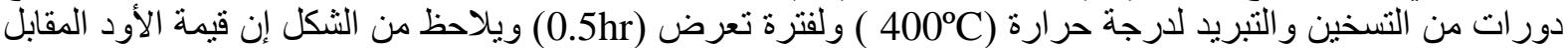

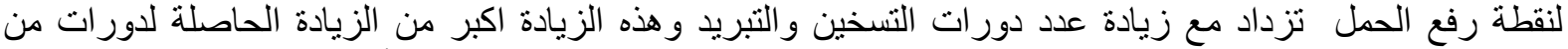

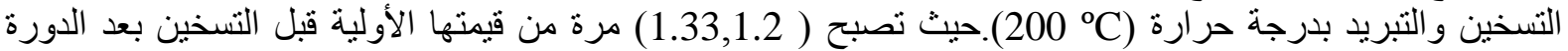
الأولى و الثانية من التسخين على التو الي.أما قيمة الحمل الأعظم فإنها تصبح \% بعد دور ات التسخين الأولى و الثانيةو الثالثة و الر ابعة على التئ التو التي.

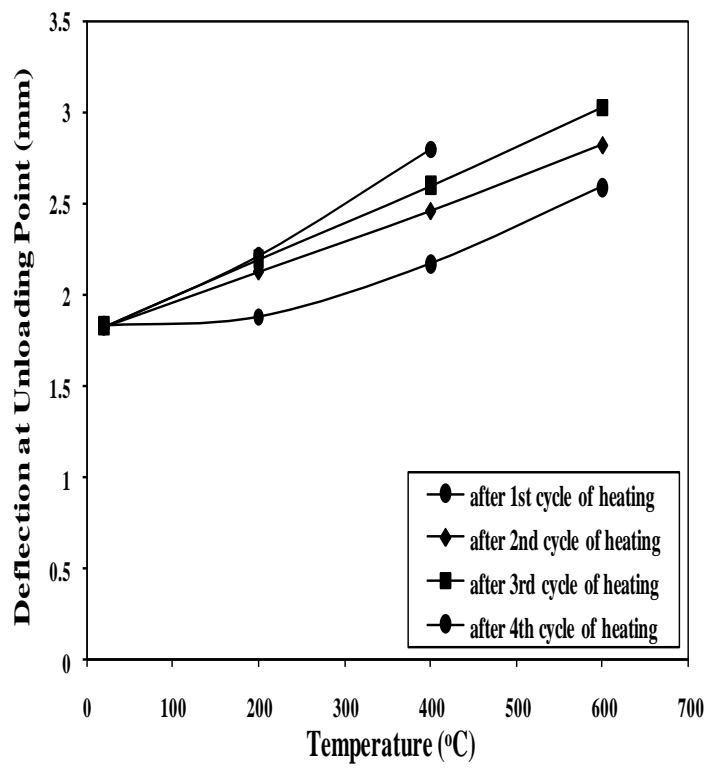

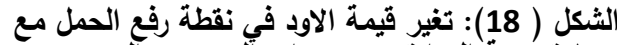

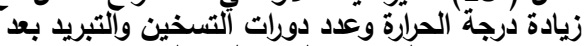
الاورة الاولى منالتحميل

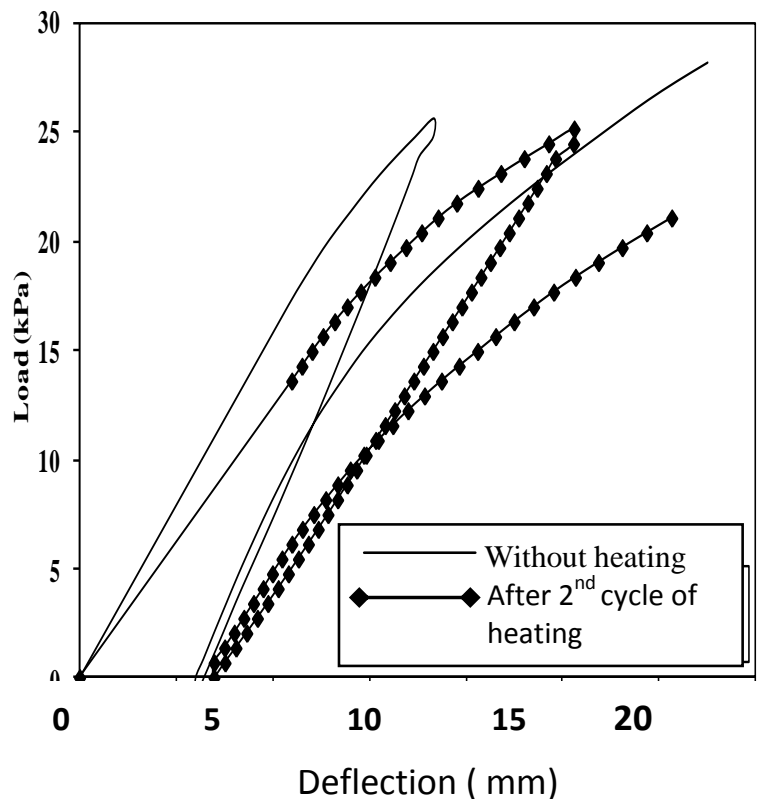

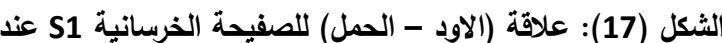

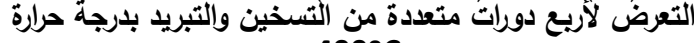
.400 으

ويمكن رسم العلاقة بين قيمة الأود في نقطة رفع الحمل ودرجةالحر ارة و عدد دورات التسخين و التبريد وكما في الثنكل

تنخفض قيمة الحمل المسبب لتشقق الصفيحة (S 1 ) مع ارتفاع درجة الحرارة وزيادة عدد دورات التسخين والتبريد

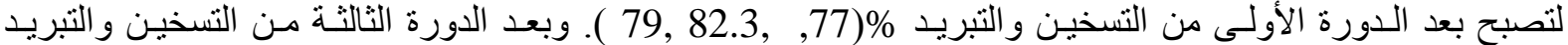
تنخفض إلى \% (8071, 75, من قيمتها الابتدائية قبل التسخين لدرجة حر ارة

ويوضح الثكل (19) الانخفاض الحاصل في الحمل المسبب للتشقق مع ارتفاع درجة الحرارة وعدد دورات التسخين

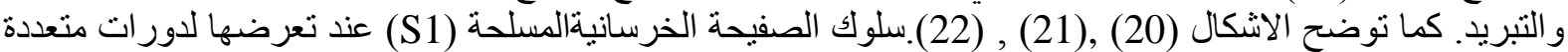

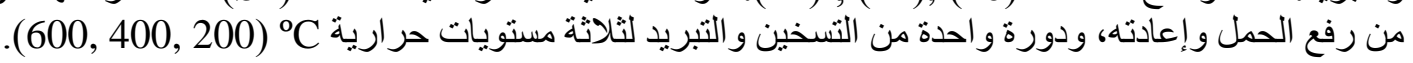




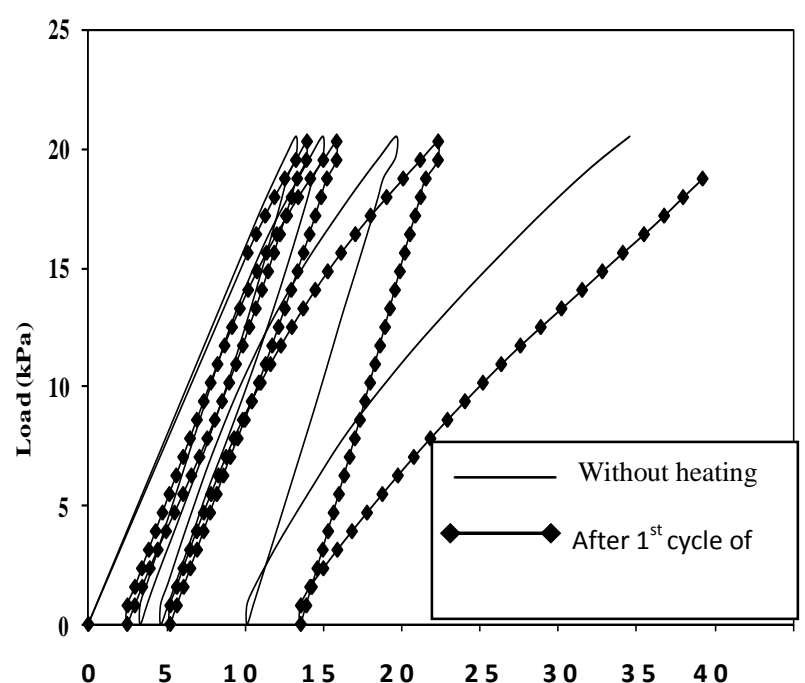

Deflection (mm)

الشكل (20)علاقة الحمل-الأود للصفيحة الخرسانية S1 بعد 200C التعرض لاورة واحدة من التسخين والتبريد بارجة حرارة

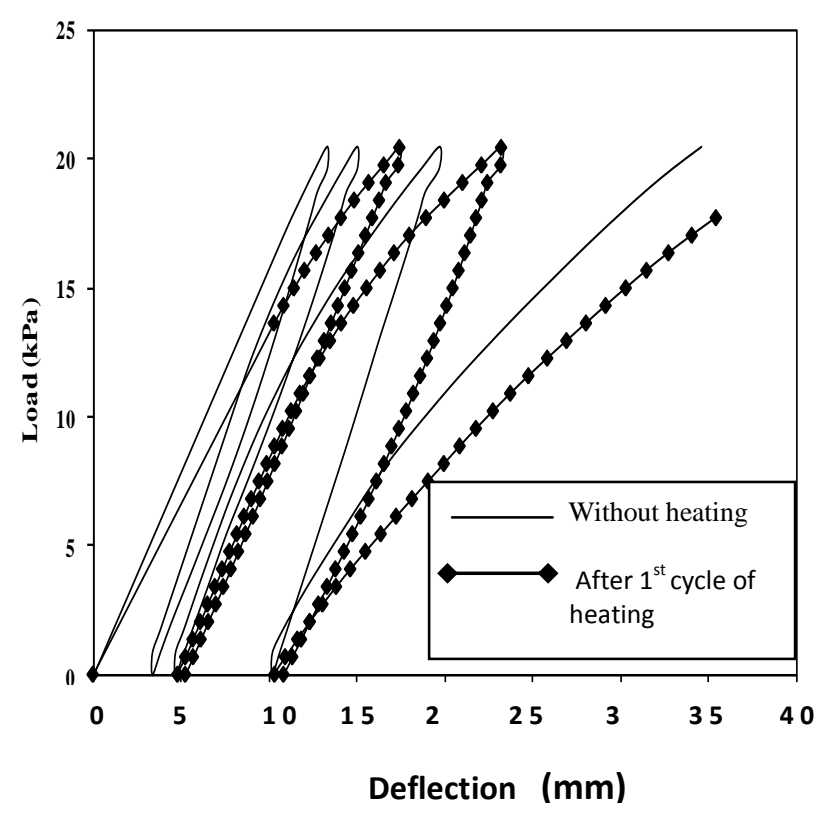

الثكل ( 22) علاقة (الاود - الحمل) للصفيحة الخرسانية

S1بعد التعرض لدورة واحدة من التسخين والتبريد بلرجة حرارة ) ( 600 ㅇ) ()

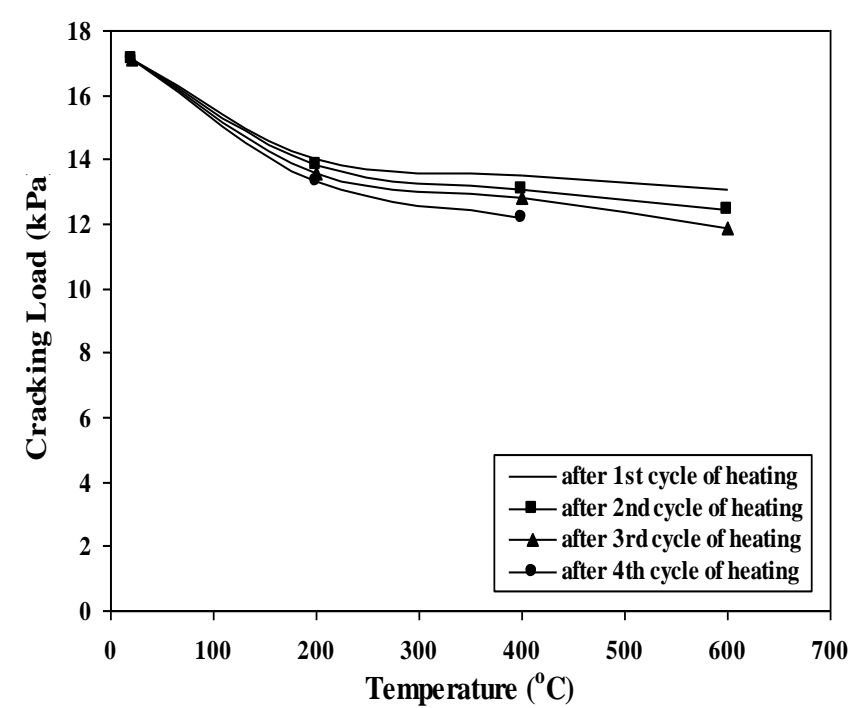

الشكل ( 19 ) : تغير قيمة حمل التشقق لصفيحة S1 مع زيادة درجة الحرارة وعدد دورات التبريد وإلتسخين

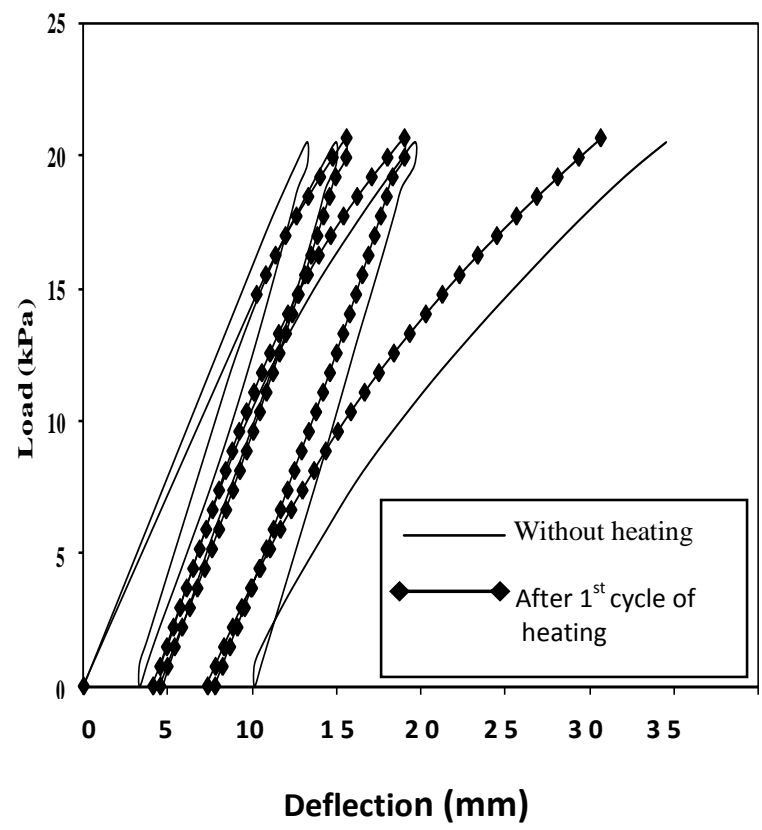

الثكل (21) علاقة (الاود - الحمل) للصفيحة الخرسانية S1 بعد التعرض لدورة واحدة من التسخين والتبريد بدرجة حرارة CC 


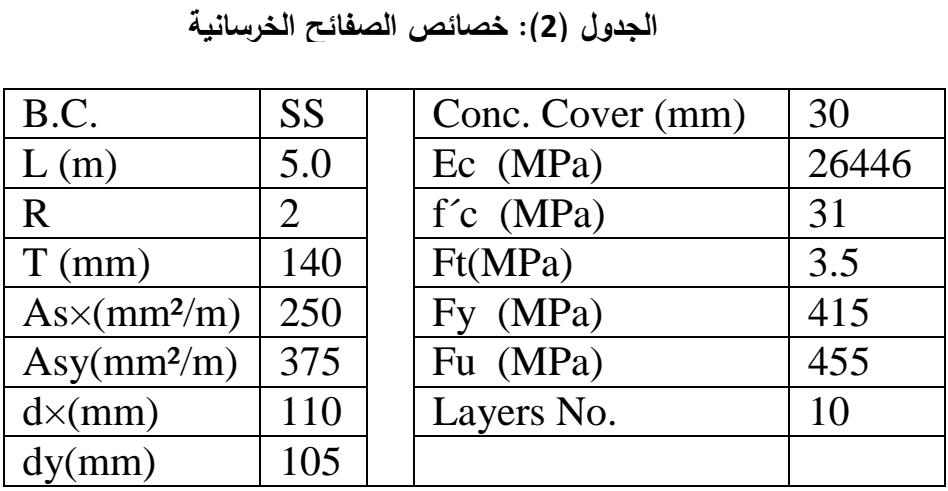

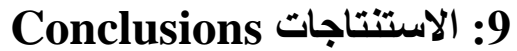

$$
\begin{aligned}
& \text { في ضوء النتائج الموضحة في الفصل السابق يمكن الخروج بالاستنتاجات الآتية: }
\end{aligned}
$$

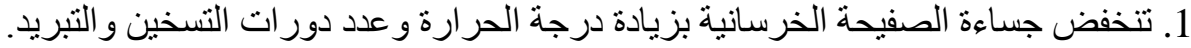

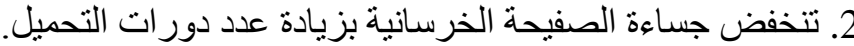

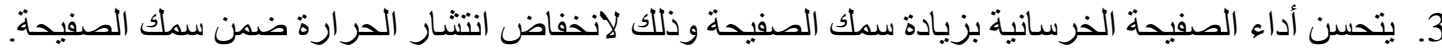

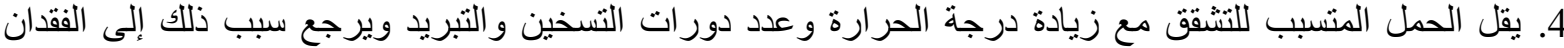

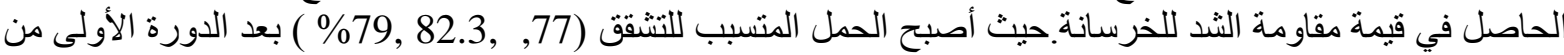

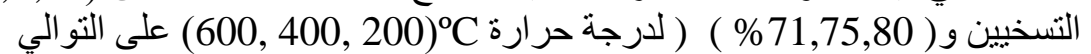

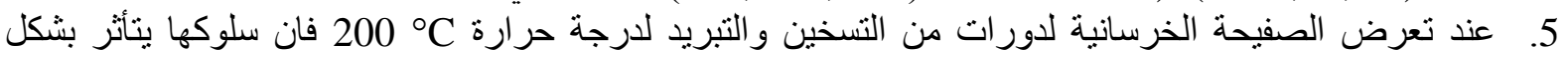

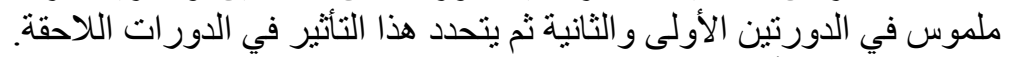

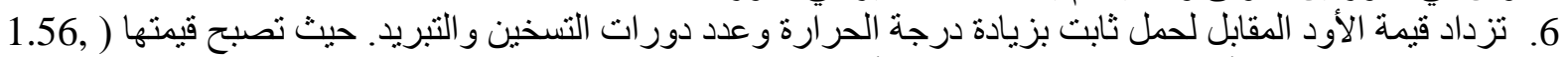
1.44) نسبة إلى قيمتها الأولية بعد دورني التسخين الأولى و الثانية على التو التي.

$$
\text { 10 ألمصادر }
$$

1. Tolentino, E., Lameris, F.S., Gomes, A., Silva, C.A. and Vasconcelos, W.L., "Effect of High Temperature on the Residual Performance of Portland Cement Concrete", Magazine of Materials Research, Vol. 5, No. 3, July 2002, pp. $301-307$.

2. Phan, L.T. and Carino, N.J., "Effect of Test Condition and Mixture Proportion on Behavior of High - Strength Concrete Exposed to High Temperature", ACI Materials Journal, Vol. 99, No. 1, January - February 2002, pp. $54-66$.

3. Cheng, F.P., Kodur, V.K.R. and Wang, T.C., "Stress - Strain Curves for High Strength Concrete at Elevated Temperatures", Journal of Material in Civil Engineering, Vol. 16, No. 1, January - February 2004, pp. $84-94$.

4. AL - Tayyib, A.J., M.H. Blauch, AL - Farabi M. Sharif and M.M. Mahamud, "The Effect of Thermal Cycling on the Durability of Concrete Model from Local Materials in the Arabian Gulf Countries", Cement and Concrete Research, Vol. 19, No. 1, 1989, pp. 131 142.

5. Otter, D.E. and Namman, A.E., "Properties of Steel Fiber Reinforced Concrete Under Cyclic Loading", ACI Material Journal, Vol. 85, No. 4, July - August 1988, pp. 254 261.

6. Otter, D.E. and Naaman, A.E., "Model for Response of Concrete to Random Compressive Loads", Journal of the Structural Division, ASCE, Vol. 115, No. 11, November 1989, pp. $2794-2809$. 
7. حسن محمد احمد، "تأثير التسخين المتناوب على الصفائح الخرسانية المسلحة السميكة"، رسالة ماجستير، جامعة الموصل 2002.

8. Bahn, B.Y. and Hsu, Cheng - Tzu, T., "Stress - Strain Behavior of Concrete Under Cyclic Loading", ACI Material Journal, Vol. 95, No. 2, March - April, 1998, pp. 178 - 193.

9. Yankelevsky, D.Z. and Reinhardt, H.W., "Uniaxial Behavior of Concrete in Cyclic tension", Journal of the Structural Division, ASCE, Vol. 115, No. 1, January 1989, pp. $166-182$.

10. Chan, E.C., "Nonlinear Geometric, Material and Time Dependent Analysis of Reinforced Concrete Shells with Edge Beam", Ph. D. Dissertation, Division of Structural Engineering and Structural Mechanics University of California, Berkley, Report No. UC - SESM 82 8, December 1982, $361 \mathrm{pp}$.

11. ACI Committee (318 - 95), "Building Code Requirements for Reinforced Concrete", American Concrete Institute, Detroit, 1983.

12. Liu, T.C.Y., Nilson, G.W. and Slate, F.O., "Biaxial Stress - Strain Relation for Concrete", Journal of the Structural Division, ASCE,Vol. 98, No. ST5, May 1972, pp. 1025 - 1034.

13. Said, A.H., "Non Linear Analysis of Reinforced Concrete Thick Plates at Elevated Temperature", M. Sc. Thesis, University of Mosul, 1999.

14. Timoshenko, S. and Woinowsky - Krieger, S., "Theory of Plates and Shells", Second Edition, McGraw - Hill, New York, 195

15. NG, S.F. and Bencharif, N., "A Finite Difference Computer Program for the Modeling of Thick Rectangular Plates”, Computer and Structures, Vol. 33, No. 4, 1989, pp. 1011 1016.

16. Rushton, N.R., "Dynamic - Relaxation Solution of Elastic - Plate Problems", Journal of Strain Analysis, Vol. 3, No. 1, 1968, pp. 23 - 32.

17. حكت علي الياس الجرجري ، " التحليل غير الخطي للصفائح الخرسـية المسلحة السميكة تحت تأثير الأحمـال

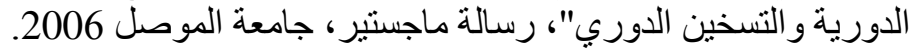

تم اجراء البحث في كلية ألهندسة = جامعة ألموصل 